\title{
Effects of Soil and Vineyard Characteristics on Volatile, Phenolic Composition and Sensory Profile of Cabernet Sauvignon Wines of Campanha Gaúcha
}

\author{
Karine P. Nicolli, ${ }^{a}$ Aline C. T. Biasoto, ${ }^{\circ b}$ Celito C. Guerra, ${ }^{c}$ Henrique P. dos Santos, ${ }^{c}$ \\ Luiz C. Correa, ${ }^{\circledR b}$ Juliane E. Welke ${ }^{\circledR d}$ and Cláudia A. Zini ${ }^{\circledR *, a}$ \\ ${ }^{a}$ Instituto de Química, Universidade Federal do Rio Grande do Sul, 91501-970 Porto Alegre-RS, Brazil \\ ${ }^{b}$ Centro de Pesquisa Agropecuária do Trópico Semi-Árido, Embrapa, Rodovia BR-428, km 152, \\ 56302-970 Petrolina-PE, Brazil \\ ${ }^{c}$ Embrapa Uva e Vinho, Rua Livramento, 515, 95701-008 Bento Gonçalves-RS, Brazil \\ ${ }^{d}$ Instituto de Ciência e Tecnologia de Alimentos, Universidade Federal do Rio Grande do Sul, \\ 91501-970 Porto Alegre-RS, Brazil
}

\begin{abstract}
The influence of vineyard on wines was evaluated for the first time using a broader analytical approach: sensory analyses and several analytical techniques (gas chromatography-mass spectrometry (GC-MS), GC flame ionization detection (FID), GC-olfactometry-Osme technique (GC-O-Osme), GC $\times$ GC-MS), taking into account odoriferous compounds of Cabernet Sauvignon wines. Sensory attributes were correlated to the concentration of volatiles and also to favorable climate/solar exposition, less clayey/lower organic matter soil, and lower vegetative growth of one out of five vineyards, C1. Among 30 coelutions in GC-MS, 12 involved some of the odoriferous volatiles and GC $\times$ GC-MS was required to elucidate their identities. Higher concentrations of ethyl propanoate and diacetyl (related with red fruits/aromatic intensity) and of acetoin (aroma of dry fruits, but coeluted with octanal in first dimension $\left({ }^{1} \mathrm{D}\right)$, which presents green odor) were found in $\mathrm{C} 1$ wine. It was also correlated with positive appearance attributes, gustatory persistence, body, smell and taste harmony, as well as with higher concentrations of phenolic acids, anthocyanins, flavonols, stilbenes, and flavanols.
\end{abstract}

Keywords: canopy management, volatile and phenolic compounds, sensorial characteristics, two-dimensional gas chromatography with mass spectrometric detection, olfactometry, liquid chromatography

\section{Introduction}

The characteristics of the vineyard, including soil type, solar orientation and canopy management (leaf removal, pruning, defoliation) can influence the sensorial and volatile composition that is related to the quality of the grapes, and consequently of the wines. ${ }^{1,2}$ Soils with low clay and organic matter contents may be adequate for the development of grapes, since these characteristics allow drainage of rainwater and can avoid a high vegetative vigor of the grapevines. The accumulation of rainwater decreases the concentration of oxygen in the soil and hinders the development of the roots. ${ }^{3}$ A high vegetative vigor impairs the development of fruits, because of the shading effect

*e-mail: cazini@iq.ufrgs.br, claudialcaraz@gmail.com on grape clusters and demands more photoassimilates to the branches than to the fruits. Vineyard characteristics involving canopy management and solar orientation are also important for grape development and their adequate control tend to provide a suitable exposure of grape clusters to solar radiation. A greater solar radiation can be achieved through a set of practices of viticulture (pruning, removal of leaves) widely used to avoid high vegetative vigor. ${ }^{4}$ According to Hunter et al., ${ }^{2}$ a greater sun exposure of the grape clusters can also be reached with the solar orientation North-South (N-S).

Scientific literature presents several reports about the consequences of the modification of vineyard characteristics on red wine quality. Feng et al. ${ }^{1}$ presented the influence of leaf removal in Pinot Noir vineyards of the Valley of Oregon on some volatiles and phenolic compounds of the corresponding wines. Two years after, they published 
a similar approach, ${ }^{5}$ but regarding five anthocyanins and 52 volatile components of USA Pinot Noir. It was also found that early leaf removal in Spanish Tempranillo vineyards influenced the composition of 34 wine volatiles compounds of the wine headspace. ${ }^{6}$ In addition, several wine volatiles of two distinct Australian sites of Cabernet Sauvignon vineyards and canopy managements were found to be different according to normalized chromatographic areas of 123 volatiles compounds that were employed for comparison. ${ }^{7}$ However, these studies are usually restricted to the evaluation of one or two field parameters (leaf removal or canopy management and site) and to one or two analytical techniques, namely gas chromatography with mass spectrometric detection (GC-MS) or comprehensive two-dimensional gas chromatography with time-of-flight MS $(\mathrm{GC} \times \mathrm{GC}$-TOFMS $)$ for the whole investigation approach. There is a lack of more comprehensive studies that would take into account several field parameters (soil type, solar orientation and canopy management) using various analytical techniques that would provide more comprehensive research results. The practice of leaf removal that increases the solar incidence on grapes has been the only one extensively studied in relation to physicochemical parameters, sensory attributes, phenolic and volatile compounds. In this case, volatiles were investigated using GC-MS and GC $\times$ GC-TOFMS, although former approaches have dealt only with semi quantitative analysis. ${ }^{1,6-8}$ In addition, statistical correlations between wine analytical parameters and canopy/field management have been rarely reported..$^{6-8}$ However, all the formerly mentioned studies have not employed GC-olfactometry (GC-O) together with multidimensional GC (MDGC) for evaluation of volatile compounds in Cabernet Sauvignon wines. A former research work of this group ${ }^{9}$ showed an extended evaluation of the quality of wines (No. of buds/ plant, No. of leaves/branch, with/without attaching shoots, irrigation, spacing between rows), using several analytical techniques (GC-O-Osme, GC-MS, GC flame ionization detector (FID) and GC $\times$ GC-TOFMS) and reported also the statistical correlations found between specific compounds and sensory characteristics, using principal component analysis (PCA), but these was performed with Merlot wines.

Cabernet Sauvignon wines from Campanha Gaúcha (Rio Grande do Sul, Brazil) are undergoing a process of geographical indication (GI) and the achievement of its denomination of origin (DO) is already envisioned as this emerging and expanding region (280 ha of vineyards in 2015) presents great potential for the production of fine wines..$^{10,11}$ A more detailed characterization of Campanha Gaúcha Cabernet Sauvignon volatiles and their relation with types of soil, canopy management and solar orientation of grapes in this region will certainly be beneficial for the processes.
It is well known that one-dimensional gas chromatography (1D-GC) can lead to errors of identification of volatile compounds due to coelutions and that GC-O is necessary to identify the active aroma volatiles. Investigations involving MDGC have been performed to evaluate some of the most potent odoriferous fractions of Shiraz wine (Australia) using a system integrated with GC-O-SNIF (surface nasal impact frequency) to relate both information, ${ }^{12}$ while the association of $\mathrm{GC} \times \mathrm{GC}$-TOFMS results have also been associated with important odoriferous regions found in two different French ciders, employing GC-O-Osme, a direct intensity method. ${ }^{13}$ However, their scope have not included a detailed characterization of the compounds of the whole volatile profile, neither the relation of this profile with results of sensory techniques, such as sensory descriptive analysis (DA). Combining chromatographic and sensory techniques is important to achieve a broader view of the quality of wines as data from GC $\times$ GC and GC-O can be related to sensory attributes of aroma (DA). ${ }^{9}$ Sensory descriptive analysis is one of the most informative tools used in the sensory evaluation of a product, as it encompasses a comprehensive description of the characteristics of aroma, appearance and flavor of a given wine and is performed by a panel of selected and trained judges, using an intensity scale. ${ }^{14}$ The association of the data obtained from several chromatographic techniques, together with sensory analysis data, potentiates the acquired knowledge and presents an innovative bias, allowing a more detailed and at the same time comprehensive view of the characterization of these wines. A previous work ${ }^{9}$ has encompassed vineyard management and aroma compounds of Merlot wines, however, correlations between Cabernet Sauvignon wine volatile and phenolic composition and sensory attributes with the type of soil, canopy management and solar orientation have not yet been investigated. The objectives of the present study were to investigate the statistical correlations among types of soil, canopy management and solar orientation of grapes of diversified types of vineyards of Campanha Gaúcha region with the sensory profile, volatile and phenolic composition of Cabernet Sauvignon wines through a wide-reaching look that combined chromatographic (GC-MS, GC-FID, GC $\times$ GC-TOFMS, GC-O, high-performance liquid chromatography (HPLC)) and DA, based on the principles of quantitative descriptive analysis (QDA).

\section{Experimental}

Reagents and chemical standards

Standard volatile compounds purchased from Aldrich (Steinheim, Germany) included: 3-methylbutanoic 
acid (isovaleric acid), hexanoic acid, octanoic acid, 1-hexanol, 1-nonanol, benzyl alcohol, 1-dodecanol, hexyl acetate, ethyl hexanoate, ethyl octanoate, ethyl decanoate, ethyl 3-methylbutanoate (ethyl isovalerate), diethyl butanedioate (diethyl succinate), 2-phenylethyl acetate, ethyl dodecanoate, 2-furanmethanol, 2-heptanone, $2(5 H)$-furanone, $\alpha$-terpineol, $\beta$-damascenone, 3 -mercaptohexanol. The purity of all listed compounds was higher than $98 \%$. Standard solutions were prepared in ethanol and diluted in a wine model solution according to previous work. ${ }^{9}$ Divinylbenzene/carboxen/polydimethylsiloxane (DVB/CAR/PDMS), 50/30 $\mu \mathrm{m}, 2 \mathrm{~cm}$, StableFlex, solid phase microextraction (SPME) fiber was purchased from Supelco (Bellefonte, USA) and conditioned according to the manufacturer recommendations. Sodium chloride $(\mathrm{NaCl})$ of analytical grade was purchased from Nuclear (São Paulo, Brazil) and oven dried at $150{ }^{\circ} \mathrm{C}$ for two hours before use. The extraction was performed in twenty milliliter headspace vials with Teflon septa purchased from Supelco (Bellefonte, USA).

Standard phenolic compounds purchased from Chem Service (West Chester, USA) included: caffeic acid, ferulic acid and gallic acid. Kaempferol-3-O-glucoside, pelargonidin-3-O-glucoside, (+)-catechin, (-)-epicatechin, $(-)$-epicatechin gallate, $(-)$-epigallocatechin gallate, isorhamnetin-3-O-glucoside, myricetin, delphinidin3-glucoside, malvidin-3-glucoside, peonidin3-O-glucoside, petunidin-3- $O$-glucoside, procyanidin A2, procyanidin $\mathrm{B} 1$, procyanidin $\mathrm{B} 2$, quercetin, quercetin3- $\beta$-D-glucoside (isoquercetin), trans-resveratrol and rutin standards were obtained from Extrasynthese (Genay, France). Chlorogenic acid and $p$-coumaric acid were purchased from Sigma-Aldrich (Gillingham, United Kingdom). Stock solutions of each standard were prepared in methanol and the solutions used to obtain the calibration curves were prepared by dilution of the respective stock solutions in $0.85 \%$ phosphoric acid solution as published elsewhere. ${ }^{15}$ Methanol, acetonitrile and phosphoric acid
85\% LC grade were supplied by Vetec Química Fina Ltda. (Rio de Janeiro, Brazil), J.T. Baker (Phillipsburg, USA) and Fluka (Geneva, Switzerland), respectively. Ultrapure water was obtained from a Purelab Option Q Elga System (High Wycombe, United Kingdom).

\section{Vineyard experimental design and wine production}

Wines produced with grapes from five vineyards (termed C1 to C5, as shown in Figure S1 (Supplementary Information (SI) section)) located in Campanha Gaúcha region, RS, Brazil were evaluated. According to Table 1, the experiments were conducted with different types of soil, canopy managements and solar orientation of rows. ${ }^{16}$ Table S1 (SI section) shows additional information about the vineyards including geographic coordinates, year of vineyard implementation, spacing between the plants and altitude. Information regarding precipitation, sunshine duration, temperature and humidity during 2013/14 in the region of Campanha Gaúcha, where the vineyards were located is presented in Table S2 (SI section). Randomly, two rows were selected in each vineyard and ten plants were demarcated along the length of each row. Cabernet Sauvignon (Vitis vinifera L.) grapes' were grafted onto $\mathrm{SO} 4$ rootstock, during the 2013/14 growth cycle. In addition, the conduction system is on trellis (espalier) for all vineyards and spacing among rows was also alike for all of them (average of $1.1 \pm 0.1 \mathrm{~m}$ among lines $\times 3.0 \pm 0.3 \mathrm{~m}$ of inter-row). The main contrasts among these vineyards were types of soil, canopy management, and solar orientation of the rows.

Approximately $80 \mathrm{~kg}$ of grapes of each vineyard were harvested on February 19, 2014 and their physicochemical characteristics are reported in Table S3 (SI section). The experimental planning was carried out in randomized blocks design and three microvinifications per vineyard were performed. Each sampling was carried out with 4 boxes of grapes of $20 \mathrm{~kg}$ each. These grapes

Table 1. Soil characteristics, canopy management and row orientation of the vineyards of the Campanha Gaúcha region, Brazil, where Cabernet Sauvignon grapes were cultivated

\begin{tabular}{|c|c|c|c|c|c|c|c|}
\hline \multirow{2}{*}{ Wine } & \multicolumn{3}{|c|}{ Soil } & \multicolumn{3}{|c|}{ Canopy management } & \multirow{2}{*}{$\begin{array}{c}\text { Orientation } \\
\text { (row) }\end{array}$} \\
\hline & Classification $^{\mathrm{a}}$ & Clay / \% & $\mathrm{OM}^{\mathrm{b}} /\left(\mathrm{g} \mathrm{kg}^{-1}\right)$ & No. bunch/plant & No. bud/plant & Ratio bunch/bud & \\
\hline $\mathrm{C} 1$ & acrisol & 14 & 11 & 35 & 30 & 1.2 & NW-SE \\
\hline $\mathrm{C} 2$ & luvisol & 27 & 24 & 37 & 20 & 1.9 & $\mathrm{~N}-\mathrm{S}$ \\
\hline $\mathrm{C} 3$ & acrisol & 20 & 17 & 28 & 20 & 1.4 & E-W \\
\hline $\mathrm{C} 4$ & luvisol & 27 & 27 & 28 & 15 & 1.9 & NE-SW \\
\hline $\mathrm{C} 5$ & acrisol & 21 & 20 & 32 & 18 & 1.8 & $\mathrm{~N}-\mathrm{S}$ \\
\hline
\end{tabular}

${ }^{a}$ The classification of soils follows the International Soil Classification System of Food and Agriculture Organization of the United Nations (IUSS Working Group WRB, 2015); ${ }^{16}$ bOM: organic material. NW: Northwest; SE: Southeast; N: North; S: South; E: East; W: West; NE: Northeast; SW: Southwest. 
were finally divided in three boxes to provide grapes for each vinification process. Wines were elaborated in the Enology Laboratory of Embrapa Grape and Wine, Bento Gonçalves, Brazil, using a traditional winemaking method for red wines. ${ }^{17}$ After harvest, grapes were stored for less than $24 \mathrm{~h}$ in a cold chamber at $10 \pm 2{ }^{\circ} \mathrm{C}$. A vertical container of stainless steel similar to those used in industrially produced wines was employed for microvinification. The winemaking was carried out after grapes were destemmed and lightly crushed, and after the addition of $80 \mathrm{mg} \mathrm{L}^{-1}$ of potassium metabisulfite $\left(\mathrm{K}_{2} \mathrm{~S}_{2} \mathrm{O}_{5}\right.$, Veneto Mercantil Importadora, Bento Gonçalves, Brazil). Alcoholic fermentation with simultaneous maceration (14 days) was performed at $25 \pm 2{ }^{\circ} \mathrm{C}$ and started after the addition of $150 \mathrm{mg} \mathrm{L}^{-1}$ of active dry yeast Saccharomyces cerevisiae bayanus (Maurivim $\mathrm{PDM}^{\circledR}$, Amazon Group, Monte Belo do Sul, Brazil). Fermentation was considered complete when density became constant and less than $0.990 \mathrm{~g} \mathrm{~mL}^{-1}$ and concentration of residual sugars was below $2 \mathrm{~g} \mathrm{~L}^{-1}$. The second fermentation (malolactic fermentation) was carried out at $18 \pm 2{ }^{\circ} \mathrm{C}$ until all lactic acid was consumed (around 40 days) and this step was monitored by paper chromatography. Wine was stored at $0{ }^{\circ} \mathrm{C}$ for six months to allow its stabilization. Before bottling, the content of sulfur dioxide was corrected to $50 \mathrm{mg} \mathrm{L}^{-1}$ of free $\mathrm{SO}_{2}$. Wines were filled into $750 \mathrm{~mL}$ dark green glass bottles and stored in an acclimatized $\left(16^{\circ} \mathrm{C}\right)$ wine cellar until their analyses. Table S3 (SI section) presents density, alcohol content, $\mathrm{pH}$, total acidity, volatile acidity, fixed acidity, reducing sugars, free $\mathrm{SO}_{2}$ and total $\mathrm{SO}_{2}$ of the five wines. These parameters were found to be according to Brazilian legislation. ${ }^{18}$

\section{Characterization of the wines sensory profile using sensory descriptive analysis}

Twelve well-experienced judges in wine sensory evaluation from the Brazilian Agricultural Research Corporation (Embrapa) evaluated the sensory profiles of Cabernet wines ( $\mathrm{C} 1$ to $\mathrm{C} 5$ ) using DA based on QDA principle,,$^{14}$ as described to Merlot wine in a previous work. ${ }^{9}$ The judges generated a consensual list with 17 attributes of appearance, aroma and taste and mouth sensations, including their definitions and references for the panel training, using Kelly's Repertory Grid Method $^{19}$ to characterize the sensory profile of wines. The wine samples were randomly presented to the judges who described the similarities and differences between the samples in appearance, aroma and taste and mouth sensations. Subsequently, the judges met and with the help of the panel leader discussed the terms mentioned in the sample description. Synonyms, antonyms and terms considered to be irrelevant were consensually grouped or eliminated, and the terms that best described the similarities and differences between the evaluated samples were selected.

The sensorial analyses procedures (DA and GC-O) were approved by the Research Ethics Committee (CEP/ UNIVASF protocol No. 1.346.299/2015 and CAAE 49561715.1.0000.5196), in compliance with Resolution 466/12, of the National Health Council, Brazil. ${ }^{20}$

\section{Determination of volatile profile}

Volatile compounds were extracted by headspace (HS-SPME) according to Welke et al. ${ }^{21}$ HS-SPME was carried out with $1 \mathrm{~mL}$ of wine in $20 \mathrm{~mL}$ glass vials, $0.3 \mathrm{~g}$ of $\mathrm{NaCl}$, at $55^{\circ} \mathrm{C}$ for $45 \mathrm{~min}$ without agitation throughout the equilibration and extraction. Volatiles were desorbed in a GC injection port at $250^{\circ} \mathrm{C}$ for $5 \mathrm{~min}$. GC $\times$ GC-TOFMS, GC-FID, GC-MS, and GC-O were used to determine the volatile profile of wines and their odoriferous compounds. Chromatographic conditions and data processing have been reported elsewhere. ${ }^{9}$ The injection, transfer line and ion source temperature were at $250{ }^{\circ} \mathrm{C}$. The oven temperature began at $35^{\circ} \mathrm{C}$ for $5 \mathrm{~min}$ and was raised to 200 at $3{ }^{\circ} \mathrm{C} \mathrm{min}^{-1}$; reaching $250{ }^{\circ} \mathrm{C}$ at $20{ }^{\circ} \mathrm{C} \mathrm{min}^{-1}$, where it was maintained for $5 \mathrm{~min}$. The secondary oven was kept $10{ }^{\circ} \mathrm{C}$ above the primary oven throughout the chromatographic run. Ultrapure helium was used as carrier gas at a constant flow of $1 \mathrm{~mL} \mathrm{~min}{ }^{-1} . \mathrm{GC} \times \mathrm{GC}$ system consisted of an Agilent 6890N (Agilent Technologies, Palo Alto, CA, USA) equipped with a Pegasus IV time-of-flight mass spectrometric detector (Leco Corporation, St. Joseph, USA). A polar column (DB-WAX, polyethylene glycol, $30 \mathrm{~m} \times 0.25 \mathrm{~mm} \times 0.25 \mathrm{~mm}$ ) and a medium-polar column (DB-17ms, 50\% phenyl, 50\% methylpolysiloxane, $1.70 \mathrm{~m} \times 0.18 \mathrm{~mm} \times 0.18 \mu \mathrm{m}$ ) was used in the first $\left({ }^{1} \mathrm{D}\right)$ and second $\left({ }^{2} \mathrm{D}\right)$ dimensions, respectively.

The odoriferous compounds of wines were determined using the Osme technique to obtain the GC-O data, as previously described in a former publication. ${ }^{9}$ In brief, the chromatographic column was disconnected from the FID of a GC (Agilent Technologies, model 6890, Palo Alto, USA) and connected to another base of the chromatograph with no detector for GC-olfactometric evaluation. A glass tube was fitted to this base, allowing the effluent of the column to be conducted through this glass tube and directed to the panelist's nose. The determination of the odoriferous compounds was performed with five judges. A consensus aromagram was built for each wine under study, averaging all peaks detected at least twice by at 
least three panelists. Odoriferous impact was measured through intensity (I) and Osme area percentage (Osme, in \%). Intensity was obtained as an average intensity of the odor of the consensual aromagram (intensity scale comprises values from 0 to 10). In addition, the area percentage corresponds to the average area of that compound analyzed by all judges.

Determination of phenolic compounds by high performance liquid chromatography-diode array detector-fluorescence detector (LC-DAD-FLD)

The analyses were performed using a Waters LC system e2695 Separation Module Alliance equipped with a quaternary solvent pump and an automatic injector. For the phenolic determination, a Waters DAD model 2998 and a Waters FLD model 2475 were employed. Data acquisition and processing were carried out using the Waters Empower $^{\mathrm{TM}} 2$ software (Milford, USA). As previously optimized by Natividade et al., ${ }^{15} 10 \mu \mathrm{L}$ of the wine were filtered through a $0.45 \mu \mathrm{m}$ nylon membrane (AllcromPhenomenex, Torrance, USA) and analyzed in a Gemini NX C-18 column $(150 \mathrm{~mm} \times 4.6 \mathrm{~mm} \times 3 \mu \mathrm{m})$ (Phenomenex) maintained at $40{ }^{\circ} \mathrm{C}$. The mobile phase consisted of a gradient mixture of solvent A $(0.85 \%$ phosphoric acid solution) and solvent B (acetonitrile), with a flow-rate of $0.5 \mathrm{~mL} \mathrm{~min}^{-1}$. FLD was used at $320 \mathrm{~nm}$ to analyze flavanols (+)-catechin, (-)-epicatechin, procyanidin A2, procyanidin $\mathrm{B} 1$, and procyanidin $\mathrm{B} 2$, while $280 \mathrm{~nm}$ encompassed (-)-epigallocatechin gallate and (-)-epicatechin gallate. Flavonols were analyzed at $360 \mathrm{~nm}$, anthocyanins at $280 \mathrm{~nm}$, phenolic acids at 320 and $280 \mathrm{~nm}$ (gallic acid) and stilbenes (trans-resveratrol) at $320 \mathrm{~nm}$.

\section{Statistical analysis}

Statistical analyses of the data were performed using SPSS version 15.0 (SPSS, Chicago). ${ }^{22}$ Analysis of variance (ANOVA) followed by the Tukey's test $(p \leq 0.05)$ was used to compare the average of the data of sensorial attributes and volatile compounds among the experiments. Spearman correlation (r) was used to determine the strength of association between attributes evaluated by DA and (i) volatile compounds identified by GC $\times$ GC-TOFMS and GC-O and (ii) phenolic compounds identified by LC-DAD-FLD. The criteria used to measure the strength of correlations among variables were: perfect $(r=1.00)$, strong $(0.80 \leq \mathrm{r}<1.00)$, moderate $(0.50 \leq \mathrm{r}<0.80)$, weak $(0.10 \leq \mathrm{r}<0.50)$, and very weak (almost none) correlation $(0.10 \leq \mathrm{r})$, according to the arbitrary scale mentioned by Granato et al..$^{23}$

\section{Results and Discussion}

Firstly, all wines (C1 to C5 as described in Table 1) were evaluated by DA, and this allowed selecting the wine that presented higher notes for positive sensorial attributes. This chosen wine was analyzed by GC-O, GC-MS and GC-FID to identify the aroma-active compounds. All wines were also analyzed by HS-SPME-GC $\times$ GC-TOFMS and the combination of all these analytical techniques resulted in a deeper elucidation of the volatile profile of Cabernet Sauvignon wines from Campanha Gaúcha, as shown in Table S4 (SI section), where compounds are numbered according to their elution order. The numbering of compounds in Table S4 is the same all over this article, including other tables.

Ten chemical classes were present, namely esters (55), alcohols (51) and terpenoids (25) present with a higher number of compounds, followed by acids (15), aldehydes (12), ketones (12), lactones (7), phenols (7), furans (7) and sulfur compounds (6), summing up 197 volatile compounds. The predominant presence of esters, alcohols and terpenoids has already been observed in a previous study ${ }^{24}$ of a South African Cabernet Sauvignon wine (60 esters, 25 alcohols and 35 terpenoids), also analyzed by HS-SPME-GC $\times$ GC-TOFMS. Robinson et al. ${ }^{7}$ employed a similar analytical approach to relate sensory attributes, field experimental conditions and wine volatiles of Australian Cabernet Sauvignon wines, although they have not performed GC-O, neither determined the concentration of wine volatiles. ${ }^{7}$ Phenylethyl alcohol (No. 63), isoamyl alcohol (3-methyl-1-butanol) (No. 27), and diethyl butanedioate (or diethyl succinate, No. 115) were major components in terms of concentration levels $(>710,>450$, $2446 \mu \mathrm{g} \mathrm{L}^{-1}$; floral, stinky, cheese for odor description, respectively, Tables 2 and S4 (SI section)) and presented great odoriferous impact on Cabernet Sauvignon wines and these same compounds stood out in Chinese Cabernet Sauvignon (58916, 4136, $1206 \mu \mathrm{g} \mathrm{L}{ }^{-1}$ to phenylethyl alcohol, isoamyl alcohol, and diethyl butanedioate, respectively).

The compounds found in wines $\mathrm{C} 1$ to $\mathrm{C} 5$ were quantified by $\mathrm{GC} \times \mathrm{GC}-\mathrm{TOFMS}$ and coelutions responsible for errors of identification related to odor compounds in GC-O (also GC-MS) were identified. The correlations between the volatile compounds evaluated by chromatographic techniques and aroma attributes described in DA were verified. Classes of phenolic compounds were evaluated by LC-DAD-FLD and then related to the appearance and taste and mouth sensations attributes evaluated by DA. Finally, the influence of the soil and vineyard characteristics was verified in relation to the sensorial attributes, volatile and phenolic compounds. 
Table 2. Volatile compounds of twenty six odoriferous regions of 2014 wines (C1-C5) identified and quantified by comprehensive two-dimensional gas chromatography with mass spectrometry detection (GC $\times$ GC-TOFMS) and described as odoriferous compounds by gas chromatography-olfactometry (GC-O). Experimental conditions are described in "Determination of volatile profile" sub-section

\begin{tabular}{|c|c|c|c|c|c|c|c|c|}
\hline $\mathrm{re}^{\mathrm{A}}$ & $\begin{array}{c}\text { Compound } \\
(\text { No. in Table S4) }\end{array}$ & $\mathrm{C} 1 /\left(\mu \mathrm{g} \mathrm{L}^{-1}\right)$ & $\mathrm{C} 2 /\left(\mu \mathrm{g} \mathrm{L}^{-1}\right)$ & $\mathrm{C} 3 /\left(\mu \mathrm{g} \mathrm{L}^{-1}\right)$ & $\mathrm{C} 4 /\left(\mu \mathrm{g} \mathrm{L}^{-1}\right)$ & $\mathrm{C} 5 /\left(\mu \mathrm{g} \mathrm{L}^{-1}\right)$ & Odor description & Odor lit ${ }^{\mathrm{C}}$ \\
\hline A & $\begin{array}{l}\text { ethyl propanoate } \\
(\text { No. } 81)^{\mathrm{a}}\end{array}$ & $11.0 \pm 1.3 \mathrm{a}$ & $7.9 \pm 1.3 \mathrm{~b}$ & $6.3 \pm 0.7 \mathrm{c}$ & $8.0 \pm 0.4 \mathrm{~b}$ & $6.3 \pm 1.3 \mathrm{c}$ & $\begin{array}{c}\text { fruity, sweet, } \\
\text { sweet fruit }\end{array}$ & sweet, fruity \\
\hline B & $\begin{array}{c}\text { diacetyl [2,3-butanedione] } \\
(\text { No. 141) }\end{array}$ & $6.4 \pm 0.4 \mathrm{a}$ & $3.9 \pm 0.6 b$ & $1.0 \pm 0.1 \mathrm{c}$ & $4.3 \pm 0.1 \mathrm{~b}$ & $1.0 \pm 0.1 \mathrm{c}$ & sweet, fruits & buttery \\
\hline $\mathrm{C}$ & $\begin{array}{l}\text { ethyl butanoate } \\
(\text { No. 86) }\end{array}$ & $9.0 \pm 1.4 \mathrm{~b}$ & $12.0 \pm 0.4 \mathrm{a}$ & $10.8 \pm 1.6 \mathrm{a}$ & $8.9 \pm 0.4 b$ & $6.9 \pm 0.7 \mathrm{c}$ & $\begin{array}{c}\text { fruity, red fruits, } \\
\text { sweet }\end{array}$ & fruity \\
\hline \multirow{2}{*}{ D } & $\begin{array}{c}\text { 1-propanol } \\
(\text { No. 18, Co1 })^{\mathrm{c}}\end{array}$ & $7.7 \pm 1.2 \mathrm{a}$ & $8.1 \pm 0.0 \mathrm{a}$ & $8.9 \pm 2.2 \mathrm{a}$ & $8.0 \pm 0.1 \mathrm{a}$ & $6.4 \pm 0.4 \mathrm{a}$ & \multirow{2}{*}{$\begin{array}{l}\text { fruity, sweet, } \\
\text { fresh }\end{array}$} & \\
\hline & $\begin{array}{c}\text { 2-butenal } \\
(\text { No. 69, Co1 })^{\mathrm{c}}\end{array}$ & $<2.0$ & $<2.0$ & $<2.0$ & $<2.0$ & $<2.0$ & & pungent \\
\hline $\mathrm{E}$ & $\begin{array}{l}\text { ethyl isovalerate } \\
\qquad \text { No. 88) }\end{array}$ & $2.0 \pm 0.4 \mathrm{~b}$ & $2.7 \pm 0.1 \mathrm{a}$ & $2.4 \pm 0.6 \mathrm{ab}$ & $2.7 \pm 0.3 \mathrm{a}$ & $1.2 \pm 0.4 \mathrm{c}$ & $\begin{array}{c}\text { fruity, ripe } \\
\text { fruit, solvent, } \\
\text { refreshing, citric }\end{array}$ & fruity \\
\hline $\mathrm{F}$ & $\begin{array}{l}\text { isobutyl alcohol } \\
(\text { No. 20) }\end{array}$ & $226.8 \pm 23.2 \mathrm{ab}$ & $204.1 \pm 33.3 \mathrm{~b}$ & $217.1 \pm 33.3 \mathrm{ab}$ & $241.5 \pm 10.1 \mathrm{ab}$ & $257.6 \pm 0.2 \mathrm{a}$ & $\begin{array}{l}\text { gas, stinky, } \\
\text { pungent, } \\
\text { herbaceous, } \\
\text { plastic } \\
\end{array}$ & $\begin{array}{l}\text { oily, bitter, } \\
\text { green }\end{array}$ \\
\hline G & $\begin{array}{l}\text { isoamyl acetate } \\
(\text { No. 90) }\end{array}$ & $87.9 \pm 18.3 \mathrm{ab}$ & $93.2 \pm 1.7 \mathrm{a}$ & $73.5 \pm 11.8 b$ & $70.3 \pm 3.1 \mathrm{~b}$ & $49.9 \pm 6.7 \mathrm{c}$ & $\begin{array}{l}\text { banana, fruits, } \\
\text { sweet, solvent, } \\
\text { acetone, stinky }\end{array}$ & $\begin{array}{l}\text { fruity, } \\
\text { banana }\end{array}$ \\
\hline $\mathrm{H}$ & $\begin{array}{l}\text { 1-butanol } \\
(\text { No. 24) }\end{array}$ & $2.0 \pm 0.5 \mathrm{c}$ & $3.9 \pm 0.7 b c$ & $2.0 \pm 0.4 \mathrm{c}$ & $6.4 \pm 1.2 \mathrm{ab}$ & $9.4 \pm 2.8 \mathrm{a}$ & fruit, dry, gas & alcoholic \\
\hline \multirow[t]{2}{*}{ I } & $\begin{array}{c}\text { 3-methyl-1-butanol } \\
\text { (isoamyl alcohol) } \\
\text { (No. 27, Co4) }^{\mathrm{c}}\end{array}$ & $>450.0$ & $>450.0$ & $>450.0$ & $>450.0$ & $>450.0$ & \multirow{2}{*}{$\begin{array}{l}\text { stinky, solvent, } \\
\text { rancid, } \\
\text { fermented, } \\
\text { vomit }\end{array}$} & solvent \\
\hline & $\begin{array}{c}\text { 2-methyl-1-butanol } \\
(\text { No. 26, Co4) }\end{array}$ & $>450.0$ & $>450.0$ & $>450.0$ & $>450.0$ & $>450.0$ & & $\begin{array}{c}\text { malt, wine, } \\
\text { onion }\end{array}$ \\
\hline $\mathrm{J}$ & $\begin{array}{l}\text { ethyl hexanoate } \\
(\text { No. 93) }\end{array}$ & $82.6 \pm 13.7 \mathrm{abc}$ & $98.8 \pm 8.3 \mathrm{a}$ & $95.7 \pm 28.0 \mathrm{ab}$ & $68.5 \pm 3.0 \mathrm{bc}$ & $55.8 \pm 4.4 \mathrm{c}$ & $\begin{array}{l}\text { fruity, sweet, } \\
\text { guarana, citric, } \\
\text { red fruits, green }\end{array}$ & fruity \\
\hline \multirow[t]{2}{*}{$\mathbf{K}$} & $\begin{array}{c}\text { acetoin [3-hydroxy- } \\
\text { 2-butanone] } \\
(\text { No. 145, Co8) }\end{array}$ & $1.4 \pm 0.1 \mathrm{a}$ & $1.2 \pm 0.2 \mathrm{ab}$ & $1.1 \pm 0.3 \mathrm{~b}$ & $1.1 \pm 0.1 \mathrm{~b}$ & $1.0 \pm 0.1 b$ & \multirow[t]{2}{*}{ green } & $\begin{array}{l}\text { creamy, } \\
\text { fatty }\end{array}$ \\
\hline & $\begin{array}{c}\text { octanal } \\
(\text { No. } 71, \text { Co8 })^{\mathrm{c}}\end{array}$ & $<2.0$ & $<2.0$ & $<2.0$ & $<2.0$ & $<2.0$ & & $\begin{array}{c}\text { green, orange, } \\
\text { juicy }\end{array}$ \\
\hline \multirow[b]{2}{*}{$\mathbf{L}$} & $\begin{array}{l}\text { ethyl octanoate } \\
(\text { No. 103, Co11 })^{\mathrm{f}}\end{array}$ & $63.1 \pm 2.3 \mathrm{c}$ & $80.5 \pm 3.1 \mathrm{~b}$ & $106.9 \pm 9.2$ a & $54.7 \pm 2.5 \mathrm{~d}$ & $61.1 \pm 5.9 \mathrm{~cd}$ & \multirow{2}{*}{$\begin{array}{c}\text { coffee, } \\
\text { unpleasant }\end{array}$} & \\
\hline & $\begin{array}{c}p \text {-cymenene } \\
(\text { No. 174, Co11 })^{g}\end{array}$ & $7.0 \pm 0.4 a$ & $6.8 \pm 0.1 \mathrm{ab}$ & $6.8 \pm 0.3 \mathrm{ab}$ & $6.6 \pm 0.1 \mathrm{~b}$ & $6.5 \pm 0.2 \mathrm{~b}$ & & $\begin{array}{l}\text { citrus, pine, } \\
\text { coffee, solvent, } \\
\text { hydrocarbon }\end{array}$ \\
\hline \multirow{3}{*}{ M } & $\begin{array}{c}\text { acetic acid } \\
(\text { No. 1, Co12 })^{\text {h }}\end{array}$ & $>2160.0$ & $>2160.0$ & $>2160.0$ & $>2160.0$ & $>2160.0$ & \multirow{3}{*}{ vinegar } & $\begin{array}{l}\text { pungent, } \\
\text { vinegar }\end{array}$ \\
\hline & $\begin{array}{c}\text { 1-heptanol } \\
(\text { No. 43, Co12) }\end{array}$ & $<2.0$ & $<2.0$ & $<2.0$ & $<2.0$ & $<2.0$ & & green \\
\hline & $\begin{array}{l}\text { isopentyl hexanoate } \\
(\text { No. 104, Co12) }\end{array}$ & $10.2 \pm 0.1 \mathrm{c}$ & $10.5 \pm 0.0 \mathrm{~b}$ & $10.8 \pm 0.1 \mathrm{a}$ & $10.2 \pm 0.0 \mathrm{c}$ & $10.2 \pm 0.0 \mathrm{c}$ & & sweet fruity \\
\hline \multirow[b]{2}{*}{$\mathbf{N}$} & $\begin{array}{l}\text { benzaldehyde } \\
\text { (No. 74, Co15) }\end{array}$ & $41.2 \pm 8.7$ ab & $41.4 \pm 3.1$ ab & $43.7 \pm 4.4 \mathrm{ab}$ & $30.9 \pm 1.7 \mathrm{~b}$ & $47.1 \pm 9.9 \mathrm{a}$ & \multirow{2}{*}{ green, leaf } & almond \\
\hline & $\begin{array}{c}\text { ethyl 2-hydroxy-butanoate } \\
(\text { No. 106, Co15) }\end{array}$ & $9.9 \pm 0.0 \mathrm{~b}$ & $10.0 \pm 0.0 \mathrm{a}$ & $10.0 \pm 0.1 \mathrm{a}$ & $10.0 \pm 0.0 \mathrm{a}$ & $9.9 \pm 0.0 \mathrm{ab}$ & & fruity, floral \\
\hline $\mathrm{O}$ & $\begin{array}{c}\text { 2,3-butanediol } \\
(\text { No. 50) }\end{array}$ & $14.6 \pm 4.1 \mathrm{a}$ & $8.6 \pm 0.5 b$ & $8.9 \pm 2.6 \mathrm{~b}$ & $7.2 \pm 0.2 \mathrm{~b}$ & $7.6 \pm 0.0 \mathrm{~b}$ & $\begin{array}{l}\text { ripe fruit, sweet, } \\
\text { fruit } \\
\end{array}$ & fruity \\
\hline $\mathrm{P}$ & $\begin{array}{l}\text { 1-octanol } \\
(\text { No. } 51)^{\mathrm{c}}\end{array}$ & $<2.0$ & $<2.0$ & $<2.0$ & $<2.0$ & $<2.0$ & fruits, sweet & fruity \\
\hline
\end{tabular}


Table 2. Volatile compounds of twenty six odoriferous regions of 2014 wines (C1-C5) identified and quantified by comprehensive two-dimensional gas chromatography with mass spectrometry detection (GC $\times$ GC-TOFMS) and described as odoriferous compounds by gas chromatography-olfactometry (GC-O). Experimental conditions are described in "Determination of volatile profile" sub-section (cont.)

\begin{tabular}{|c|c|c|c|c|c|c|c|c|}
\hline $\mathrm{re}^{\mathrm{A}}$ & $\begin{array}{c}\text { Compound } \\
(\text { No. in Table S4) }\end{array}$ & $\mathrm{C} 1 /\left(\mu \mathrm{g} \mathrm{L}^{-1}\right)$ & $\mathrm{C} 2 /\left(\mu \mathrm{g} \mathrm{L}^{-1}\right)$ & $\mathrm{C} 3 /\left(\mu \mathrm{g} \mathrm{L}^{-1}\right)$ & $\mathrm{C} 4 /\left(\mu \mathrm{g} \mathrm{L}^{-1}\right)$ & $\mathrm{C} 5 /\left(\mu \mathrm{g} \mathrm{L}^{-1}\right)$ & Odor description & Odor lit $^{\mathrm{C}}$ \\
\hline \multirow[t]{2}{*}{$\mathbf{Q}$} & $\begin{array}{l}\gamma \text {-butyrolactone } \\
(\text { No. 154, Co18 })^{j}\end{array}$ & $366.4 \pm 102.0 \mathrm{~d}$ & $569.5 \pm 112.5$ bc & $468.7 \pm 135.0 \mathrm{~cd}$ & $639.8 \pm 51.2 \mathrm{ab}$ & $758.1 \pm 26.1 \mathrm{a}$ & \multirow{2}{*}{$\begin{array}{c}\text { foot odor, } \\
\text { stinky, rancid, } \\
\text { cheese, cooking } \\
\text { gas, fermented }\end{array}$} & $\begin{array}{l}\text { caramel, } \\
\text { sweet, } \\
\text { coconut, } \\
\text { cheese }\end{array}$ \\
\hline & $\begin{array}{l}\text { ethyl 2-furoate } \\
\text { (No. 138, Co18) }^{\mathrm{i}}\end{array}$ & $21.4 \pm 3.6 \mathrm{c}$ & $46.2 \pm 6.1 \mathrm{a}$ & $6.0 \pm 0.0 \mathrm{~d}$ & $47.9 \pm 5.4 \mathrm{a}$ & $35.4 \pm 9.5 \mathrm{~b}$ & & balsamic \\
\hline \multirow{3}{*}{$\mathbf{R}$} & $\begin{array}{l}\text { ethyl decanoate } \\
(\text { No. 112, Co19) }\end{array}$ & $60.9 \pm 5.6 \mathrm{~b}$ & $64.5 \pm 1.5 b$ & $77.5 \pm 5.1 \mathrm{a}$ & $43.5 \pm 4.0 \mathrm{~d}$ & $55.5 \pm 5.6 \mathrm{c}$ & \multirow{3}{*}{$\begin{array}{l}\text { honey, sweet, } \\
\text { rancid, acid }\end{array}$} & $\begin{array}{c}\text { fruity, grape, } \\
\text { sweet }\end{array}$ \\
\hline & $\begin{array}{l}\text { butanoic acid } \\
(\text { No. 4, Co19) }\end{array}$ & $33.7 \pm 3.6 \mathrm{ab}$ & $27.9 \pm 8.0$ bc & $32.8 \pm 9.0 \mathrm{ab}$ & $38.5 \pm 0.9 \mathrm{a}$ & $19.2 \pm 5.5 \mathrm{c}$ & & rancid \\
\hline & $\begin{array}{c}\text { menthol } \\
(\text { No. 178, Co19) }\end{array}$ & $5.7 \pm 0.0 \mathrm{a}$ & $5.7 \pm 0.0 \mathrm{a}$ & $5.8 \pm 0.1 \mathrm{a}$ & $5.7 \pm 0.0 \mathrm{a}$ & $5.7 \pm 0.0 \mathrm{a}$ & & peppermint \\
\hline \multirow[t]{2}{*}{$\mathbf{S}$} & $\begin{array}{l}\text { diethyl butanedioate } \\
\text { [diethyl succinate] } \\
(\text { No. 115, Co21) }\end{array}$ & $2466.0 \pm 65.7$ a & $748.0 \pm 13.6 \mathrm{~b}$ & $961.2 \pm 238.2 \mathrm{~b}$ & $702.9 \pm 0.7 \mathrm{~b}$ & $779.3 \pm 31.5 b$ & \multirow{2}{*}{$\begin{array}{c}\text { stinky, } \\
\text { cheese, foot } \\
\text { odor, rancid, } \\
\text { fermented, } \\
\text { vomit }\end{array}$} & $\begin{array}{c}\text { faint, } \\
\text { pleasant }\end{array}$ \\
\hline & $\begin{array}{c}\text { isovaleric acid } \\
(\text { No. 5, Co21 })^{\mathrm{u}}\end{array}$ & $580.1 \pm 43.7$ a & $569.4 \pm 35.8$ a & $573.8 \pm 12.5$ a & $582.2 \pm 51.2 \mathrm{a}$ & $599.3 \pm 24.8$ a & & $\begin{array}{c}\text { cheesy, } \\
\text { herbaceous }\end{array}$ \\
\hline \multirow{4}{*}{$\mathbf{T}$} & $\begin{array}{l}\text { 3-(methylthio)-1-propanol } \\
(\text { No. 196, Co22) }\end{array}$ & $13.1 \pm 2.5 \mathrm{~b}$ & $20.5 \pm 2.0 \mathrm{a}$ & $9.5 \pm 2.0 \mathrm{~d}$ & $12.0 \pm 1.9 b c$ & $11.7 \pm 3.0 \mathrm{c}$ & \multirow{4}{*}{$\begin{array}{l}\text { rancid, cooked, } \\
\text { legume, green, } \\
\text { gas, sweet, } \\
\text { artificial }\end{array}$} & $\begin{array}{c}\text { boiled } \\
\text { cabbage }\end{array}$ \\
\hline & $\begin{array}{l}\text { (6Z)-nonen-1-ol } \\
\text { (No. 56, Co22) }\end{array}$ & $<1.9$ & $<1.9$ & $<1.9$ & $<1.9$ & $<1.9$ & & melon \\
\hline & $\begin{array}{c}\text { 2-undecanol } \\
(\text { No. 57, Co22) }\end{array}$ & $4.3 \pm 0.1 \mathrm{a}$ & $3.17 \pm 0.1 \mathrm{~b}$ & $4.0 \pm 0.3 \mathrm{a}$ & $2.8 \pm 0.1 \mathrm{c}$ & $3.3 \pm 0.2 \mathrm{~b}$ & & $\begin{array}{c}\text { minty, fresh } \\
\text { flavors }\end{array}$ \\
\hline & $\begin{array}{c}\text { isopiperitone } \\
(\text { No. 180, Co22) }\end{array}$ & $5.7 \pm 0.1 \mathrm{a}$ & $5.7 \pm 0.1 \mathrm{a}$ & $5.7 \pm 0.1 \mathrm{a}$ & $5.8 \pm 0.1 \mathrm{a}$ & $5.7 \pm 0.1 \mathrm{a}$ & & $\operatorname{mint}$ \\
\hline $\mathrm{U}$ & $\begin{array}{c}\text { 1,2-dihydro-1,1,6-trimethyl- } \\
\text { naphthalene [TDN] } \\
(\text { No. 183) }\end{array}$ & $14.3 \pm 0.5 \mathrm{bc}$ & $14.3 \pm 0.7 b c$ & $15.0 \pm 0.6 b$ & $13.6 \pm 1.6 \mathrm{c}$ & $16.5 \pm 0.7 \mathrm{a}$ & $\begin{array}{l}\text { rancid, stinky, } \\
\text { rotten }\end{array}$ & $\begin{array}{c}\text { petrolly } \\
\text { kerosene-like } \\
\text { aroma }\end{array}$ \\
\hline V & $\begin{array}{l}\text { diethyl pentanedioate } \\
(\text { No. 119) }\end{array}$ & $10.9 \pm 0.1 \mathrm{~b}$ & $10.9 \pm 0.1 \mathrm{ab}$ & $10.9 \pm 0.1 \mathrm{ab}$ & $11.0 \pm 0.1 \mathrm{a}$ & $10.9 \pm 0.1 \mathrm{ab}$ & $\begin{array}{l}\text { pungent, } \\
\text { cooking gas, } \\
\text { fresh }\end{array}$ & $\mathrm{nf}$ \\
\hline \multirow[t]{2}{*}{ W } & $\begin{array}{l}\text { 2-phenylethyl acetate } \\
\text { (No. 125, Co26) }\end{array}$ & $5.0 \pm 0.1 \mathrm{a}$ & $2.2 \pm 0.6 \mathrm{c}$ & $2.6 \pm 0.0 \mathrm{bc}$ & $3.1 \pm 0.1 \mathrm{~b}$ & $3.3 \pm 0.1 \mathrm{~b}$ & \multirow{2}{*}{$\begin{array}{l}\text { floral, roses, } \\
\text { jasmine }\end{array}$} & $\begin{array}{l}\text { floral, rose, } \\
\text { sweet, honey, } \\
\text { fruity }\end{array}$ \\
\hline & $\begin{array}{l}\beta \text {-damascenone } \\
(\text { No. 187, Co26) }\end{array}$ & $47.3 \pm 3.7 \mathrm{a}$ & $36.7 \pm 0.4$ bc & $41.2 \pm 2.3 \mathrm{~b}$ & $33.5 \pm 0.0 \mathrm{c}$ & $34.1 \pm 0.1 \mathrm{c}$ & & $\begin{array}{l}\text { sweet, fruity, } \\
\text { rose-like }\end{array}$ \\
\hline \multirow{2}{*}{$\mathbf{X}$} & $\begin{array}{l}\text { phenylethyl alcohol } \\
(\text { No. 63, Co29) }\end{array}$ & $>710.0$ & $>710.0$ & $>710.0$ & $>710.0$ & $>710.0$ & \multirow{2}{*}{$\begin{array}{l}\text { floral, perfume, } \\
\text { sweet, jam, } \\
\text { green, acid }\end{array}$} & roses, honey \\
\hline & $\begin{array}{c}\gamma \text {-octalactone } \\
(\text { No. 156, Co29) } \\
\end{array}$ & $281.7 \pm 64.6 \mathrm{a}$ & $308.6 \pm 2.4$ a & $288.3 \pm 53.4$ a & $319.7 \pm 1.8$ a & $274.2 \pm 11.4 \mathrm{a}$ & & coconut \\
\hline $\mathrm{Y}$ & $\begin{array}{l}\text { 1-dodecanol } \\
(\text { No. 64) }\end{array}$ & $24.7 \pm 0.0 \mathrm{a}$ & $24.9 \pm 0.0 \mathrm{a}$ & $25.1 \pm 0.4 \mathrm{a}$ & $24.9 \pm 0.1 \mathrm{a}$ & $24.8 \pm 0.0 \mathrm{a}$ & $\begin{array}{c}\text { rubber bullet, } \\
\text { sweet }\end{array}$ & floral \\
\hline Z & $\begin{array}{l}\text { octanoic acid } \\
(\text { No. } 11)^{1}\end{array}$ & $>540.0$ & $>540.0$ & $>540.0$ & $>540.0$ & $>540.0$ & coffee, grain & fatty, rancid \\
\hline
\end{tabular}

Capital letters designate the 26 odoriferous regions that encompass one or more compounds. After the name of the compound, the number reported is according to Table S4 and the coelution designation is also named after Table S4 (Cox, where $\mathrm{x}$ is the coelution number also according to Table S4). In the same line means showing common letter are not significantly different $(p=5 \%)$ according to ANOVA and Tukey's test. Are: designation of odoriferous regions with capital letters, according to GC-O results for $\mathrm{C} 1$ wine; ${ }^{\mathrm{B}}$ coeluting compounds are in bold letters; quantification was performed with external calibration curves for the following compounds: ${ }^{\mathrm{a} e t h y l ~ h e x a n o a t e ; ~}{ }^{\mathrm{b}}$-heptanone; ${ }^{\mathrm{c}} 1$-hexanol; ${ }^{\mathrm{d}}$ ethyl isopentanoate; ${ }^{\mathrm{e}}$ hexyl acetate; ${ }^{\mathrm{f}}$ ethyl octanoate; ${ }^{\mathrm{g}} \alpha$-terpineol; ${ }^{\mathrm{h}} \mathrm{hexanoic}$ acid;

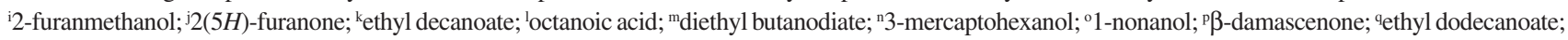
r2-phenylethyl acetate; 'benzyl alcohol; '1-dodecanol; "isovaleric acid; Cliterature sources for odors are listed in the Table S4. nf: not found. 
Sensory evaluation and determination of the odoriferous compounds

Results of DA for aroma, appearance and taste and mouth sensations attributed to wines ( $\mathrm{C} 1$ to $\mathrm{C} 5)$ are shown in Figure 1. Table S5 (SI section) presents mean scores (scale from zero to nine, according to "Characterization of the wines sensory profile using sensory descriptive analysis" sub-section) of the evaluation of judges for each DA sensory attribute. ANOVA and Tukey's test indicated that the notes attributed to undesirable aroma and vegetal aroma, sweetness and sourness did not have significant difference $(p>0.05)$ among the samples evaluated.

Several higher notes were given to positive attributes of $\mathrm{C} 1$ sample if compared to one or more of the other wine samples. Aromatic intensity, aroma of red fruits, alcoholic aroma (compared to C3/C5); spices (compared to C3); aroma of dry fruits (compared to C5), as well as in regards to appearance (color intensity and red-purple tonality compared to C2-C5) and also concerning taste and mouth sensation attributes (gustatory persistence and body compared to C2, C3, C5), smell and taste harmony (compared to C3/C5), astringency and bitterness (compared to $\mathrm{C} 3$ ). Furthermore, the negative attribute of herbaceous aroma was present in less intensity in $\mathrm{C} 1$ wine with significant difference in relation to $\mathrm{C} 3$ wine. However, the interpretation of the benefits of dissimilarities of these sensory attributes demands further investigation as the final aim is to achieve a balance among them and other wine characteristics. On the basis of the positive sensorial characteristics of the $\mathrm{C} 1$ wine, this sample was selected for GC-O-Osme analysis.

Twenty-six odoriferous chromatographic regions were found in $\mathrm{C} 1$ wine evaluated by GC-O and among them, $56 \%$ contribute positively to aroma, imparting mainly notes described as fruity, floral, sweet and others. Table S6 (SI section) shows the identification of 26 odorous volatiles of $\mathrm{C} 1$ wine (in ascending order of retention indices), according to GC-MS, in addition to the

(a)

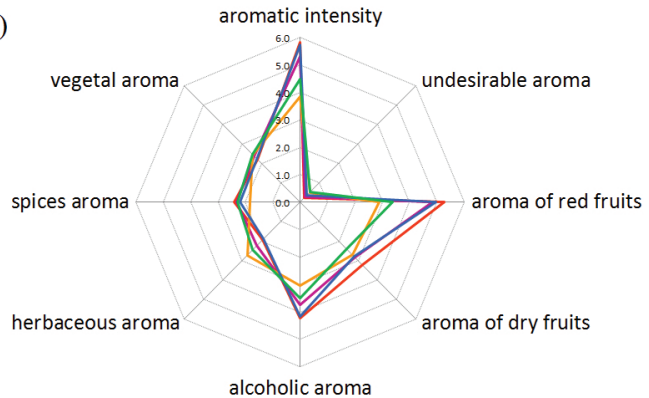

intensity (I) of the odor perceived in the effluent and the percentage of odoriferous peak area relative to total area of GC-O-Osme aromagram. These same 26 odoriferous regions detected in $\mathrm{C} 1$ wine were also found in other wines ( $\mathrm{C} 2$ to $\mathrm{C} 5$ ) analyzed through $\mathrm{GC} \times \mathrm{GC}$, covering 42 compounds ( 26 odoriferous +16 non-odoriferous). A careful inspection pointed that, among them, 12 regions included chromatographic coelutions in ${ }^{1} \mathrm{D}$, encompassing 28 compounds. These coeluting volatiles were correctly identified only after separation in the second chromatographic dimension $\left({ }^{2} \mathrm{D}\right)$ and they are shown in bold letters in Table 2 .

In total, 65 compounds were involved in 30 coelutions and they have been numbered from Co1 to Co30, as indicated between brackets after the name of the compounds, in Table S4 (SI section) and others. "Co" stands for the word "coeluting" and compounds that present the same "Co" number are the ones involved in this specific coelution. Of the 12 coelutions involving odoriferous compounds (Table 2), six resulted in odoriferous regions (D, K, L, N, W, X) that positively contributed to wine quality, other three coelutions (odoriferous regions $\mathrm{M}, \mathrm{Q}$, S) showed negative contribution and other three coelutions presented antagonistic alternate contributions (regions I, $\mathrm{R}$ and $\mathrm{T}$ ). Table S4 (SI section) presents references of all literature sources regarding odor of every single compound discussed in this section.

Seven compounds showed greater odoriferous impact in $\mathrm{C} 1$ wine, which was measured through I and Osme (in \%), as shown in Tables 2 and S6 (SI section): 2-phenylethyl acetate (W, described as floral, roses, I = 7.3; Osme $=10.5 \%$ ), phenylethyl alcohol (odoriferous region $X$, described as floral, $\mathrm{I}=5.5$; Osme $=5.5 \%$ ), ethyl hexanoate (region $\mathrm{J}$, described as fruity, $\mathrm{I}=5.6$; Osme $=4.3 \%$ ), with positive contribution and diethyl butanedioate ( $\mathrm{S}$, described as stinky, cheese, $\mathrm{I}=6.9$; Osme $=9.1 \%$ ), and acetic acid $(\mathrm{M}$, described as vinegar, $\mathrm{I}=6.9 ;$ Osme $=8.5 \%)$ with negative contribution. Isoamyl alcohol (3-methyl-



Figure 1. Mean scores of 17 sensory attributes generated through DA for Cabernet Sauvignon wines produced in 2014 using grapes grown in different wineries of the Campanha Gaúcha (C1 to C5 as shown in Table 1): (a) aroma attributes (aromatic intensity, undesirable aroma, aroma of red fruits, aroma of dry fruits, alcoholic aroma, herbaceous aroma, spices aroma and vegetal aroma) and (b) appearance (color intensity, red-purple tonality) and taste and mouth sensations attributes (gustatory persistence, sourness, bitterness, sweetness, astringency, body, smell and taste harmony). 
1-butanol $)(\mathrm{I}=5.9$; Osme $=6.6 \%)$ of region I was defined with alternate negative and positive attributes: stinky/fruity. Isoamyl alcohol has been reported as solvent and 2-methyl1-butanol as malt, wine, onion, and both tend to present a fusel odor. Interestingly, a description of fruity/banana is given to isoamyl alcohol by The Good Scent Company ${ }^{25}$ and similar odor definition is mentioned when this alcohol is produced along with other esters through de novo synthesis (Table S4, SI section).

Another important contribution corresponds to region $\mathrm{T}$, where antagonistic sensory effects were perceived by the examiners. The compound identified by GC-MS as 3-methylthio-1-propanol $(\mathrm{I}=5.4$; Osme $=4.9 \%)$ lends a cooked, legume odor, according to Tables 2 and S6 (SI section) and coeluted with three other components: ((6Z)-nonen-1-ol, 2-undecanol, and isopiperitone)) that positively contribute to aroma and might be linked to the designation of green and sweet odors of the olfactometric panel. Even though these last three compounds were present in lower concentrations than the sulfur compound, their presence was also perceived. This was also the case of odoriferous region $\mathrm{R}$, where ethyl decanoate was mistaken in GC-MS as responsible for the honey/sweet and rancid alternate odors sensed by the judges. GC $\times$ GC-TOFMS analyses have shown a partial coelution of ethyl decanoate $\left({ }^{1} t_{R}\right.$ (retention time in the first dimension $)=34.18 \mathrm{~min}$, ${ }^{2} \mathrm{t}_{\mathrm{R}}$ (retention time in the second dimension $)=5.48 \mathrm{~s}$, fruity, grape, sweet) with butanoic acid $\left({ }^{1} t_{R}=34.07 \mathrm{~min},{ }^{2} t_{R}=1.82 \mathrm{~s}\right.$, rancid odor) and menthol $\left({ }^{1} t_{R}=34.18 \mathrm{~min},{ }^{2} t_{R}=3.21 \mathrm{~s}\right.$, peppermint aroma) in ${ }^{1} \mathrm{D}$. The information provided by $\mathrm{GC} \times \mathrm{GC}$ were essential for the correct interpretation of the switching odors in chromatographic eluates. Apparently, the aromatic contribution of menthol was suppressed, as it was not perceived by the olfactometric panel.

Phenomena such as suppression and synergism of odors might have happened in several odoriferous regions due to different types of coelutions. For example, it seems that the odor of phenylethyl alcohol (roses, honey) mixed with $\gamma$-octalactone (coconut) gave rise to a different perception for region $\mathrm{X}$ from the evaluators' point of view: floral, perfume, sweet, jam and green. Furthermore, some other regions that presented positive influence on wine may be mentioned. Description of region D (fruity, sweet, fresh) matches with the odor found for 1-propanol (fruity) and the one of the coeluting 2-butenal (pungent), as their mixture may have given rise to the olfactometric panel description. Also, the interaction of benzaldehyde (almonds) and ethyl3-hydroxy-butanoate (fruity, floral) in region $\mathrm{N}$ produced a different sense of green, leaf aroma.

$\gamma$-Butyrolactone of region Q is reported as caramel, coconut, sweet, but also as cheese-like. As the coeluting compound (ethyl-2-furoate) has been described as balsamic, it seems that a combination of both compounds resulted in a negative mixed perception for the judges (foot odor, stinky, rancid, cheese, cooking gas, fermented). On the other hand, region $\mathrm{W}$ seems to present a combination of the odors of 2-phenylethyl acetate (floral, rose, sweet, honey, fruity) and of the coeluting isoprenoid ( $\beta$-damascenone, sweet, fruity, rose-like) which is reported to have a variable odor. It seems that the floral aspect of the scents of both compounds was prevalent because the judges classified it as floral, but not as fruity. The suppression of 1-heptanol (green) and isopentyl hexanoate (sweet fruity) in region M by acetic acid has probably happened, as the examiners recognized only a vinegar odor. Identification of diethyl butanedioate was done by GC-MS and it was pointed as the compound responsible for a cheese odor by GC-O-Osme (region $\mathrm{S}$ ). This ester $\left({ }^{1} \mathrm{t}_{\mathrm{R}}=35.70 \mathrm{~min}\right.$ and ${ }^{2} \mathrm{t}_{\mathrm{R}}=3.38 \mathrm{~s}$ ) is reported in the literature as presenting a pleasant aroma, according to Table 2. However, it coeluted with isovaleric $\operatorname{acid}\left({ }^{1} t_{R}=35.58 \mathrm{~min},{ }^{2} t_{R}=1.87 \mathrm{~s}\right)$ and its cheesy, herbaceous odor prevailed over the aroma of the ester, resulting in the following description of odor: stinky, cheese, foot odor, rancid, fermented, vomit. Diethyl butanedioate (2466.0 $\mu \mathrm{g} \mathrm{L}^{-1}$ ) presented higher concentration than 3-methylbutanoic acid $\left(580.1 \mu \mathrm{g} \mathrm{L}^{-1}\right)$ in $\mathrm{C} 1$ wine and this has also happened to all wine samples. Therefore, the ester peak may visually have hidden the peak of the isovaleric acid in the GC-MS chromatogram. This is a clear example that the intensity of an olfactory sensation does not depend only on the concentration of a volatile compound, but it is also influenced by several other parameters, such as volatility, solubility and vapor pressure, resulting in different partition coefficients and consequently, different concentrations in wine headspace. ${ }^{26}$ This same coelution and further separation in ${ }^{2} \mathrm{D}$ has been already mentioned for Merlot wine headspace, but its peculiar characteristics had not yet been discussed. ${ }^{9}$

Acetoin $\left({ }^{1} t_{R}=19.37\right.$ min and ${ }^{2} t_{R}=2.18 \mathrm{~s}$, creamy, fatty, Tables 2 and S4 (SI section)) and octanal $\left({ }^{1} t_{R}=19.37 \mathrm{~min}\right.$ and ${ }^{2} t_{R}=4.26 \mathrm{~s}$, green, orange, juicy, Tables 2 and $\mathrm{S} 4$ ) eluted together (region $\mathrm{K}$ ) and apparently octanal has also suppressed the aroma of acetoin as the judges found green as a resulting scent. Even though octanal concentration was below limit of detection (LOD $<2.0$ ), its green odor has prevailed over the aroma of acetoin (creamy, fatty). Acetoin is generally presented as a positive contribution and in the next section, a high correlation between this hydroxy ketone and the aroma of dry fruits will be presented for Campanha Gaúcha Cabernet Sauvignon wine, which means that in the presence of octanal, the investigation of its aroma in wine poses a difficult challenge to 1D-GC. This coelution (Co8) 
is being reported for the first time in wine headspace. The "green" contribution of octanal was clearly linked to the freshness of orange and juicy scents, and it has already been associated with pleasant aromas in wine when present in lower concentrations (Table S4, SI section).

Similar phenomenon of suppression might have happened in region L (coffee, unpleasant), where $p$-cymenene (solvent, hydrocarbon, citrus, pine, coffee) surpassed the odor of the coeluting ester ethyl octanoate (fruity). $p$-Cymenene is part of coffee aroma-presenting a gassy, styrene like odor, when undiluted. It has also been presented as solvent and hydrocarbon, although it has been defined as citrusy lemon like when diluted (Table S4, SI section). In fact, the judges mentioned coffee and also unpleasant odor which might be linked to $p$-cymenene. Despite the "unpleasant" adjective, this odoriferous region was reported as a positive contribution to wine aroma due to the "coffee" description, which is considered favorable to red wines.

\section{Correlation between aroma attributes and volatile compounds}

Odoriferous volatiles (including the ones involved in coelutions in Table 2) and their aroma attributes (Table S5, SI section) were related according to Spearman correlation in Table 3, which is represented by " $r$ ". Some compounds showed high and positive relationship with aroma attributes according to the arbitrary scale defined by Granato et al. ${ }^{23}$ including aromatic intensity and aroma of red fruits $(\mathrm{C} 1, \mathrm{C} 2$ and C4, Table S5) with ethyl propanoate (region A, Table 2, fruity) and diacetyl (2,3-butanedione, region B, sweet and fruity, $\mathrm{r}$ for both compounds: 0.98 , Table 3 ). According to Table 2, concentration of ethyl propanoate $\left(11.0 \mu \mathrm{g} \mathrm{L} \mathrm{L}^{-1}\right)$ is higher in $\mathrm{C} 1\left(11.0 \mu \mathrm{g} \mathrm{L}^{-1}\right), \mathrm{C} 2 / \mathrm{C} 4\left(7.9 / 8.0 \mu \mathrm{g} \mathrm{L}^{-1}\right)$ than in $\mathrm{C} 3 / \mathrm{C} 5\left(6.3 \mu \mathrm{g} \mathrm{L}^{-1}\right)$. A similar profile is seen for diacetyl. These same compounds also showed high and positive correlation with alcoholic and dry fruits aroma ( $\mathrm{r}$ for both compounds $=0.95$ ), although DA results have shown significant differences between $\mathrm{C} 1, \mathrm{C} 2, \mathrm{C} 4$ and $\mathrm{C} 3 / \mathrm{C} 5$ for alcoholic aroma and between $\mathrm{C} 1-\mathrm{C} 4$ and $\mathrm{C} 5$ for aroma of dry fruits (Table S5, SI section). Interestingly, results of a study ${ }^{27}$ about the acceptability of red wines produced from Vitis labrusca and hybrid grapes encompassing 120 consumers has shown that consumers acceptance increased with notes of alcohol aroma. In fact, C2 and C4 presented an average behavior among all wine samples and the greatest differences have been found among $\mathrm{C} 1$ and $\mathrm{C} 3 / \mathrm{C} 5$ (in relation to aroma of red fruits, aromatic intensity, alcoholic aroma and regarding concentrations of diacetyl and ethyl propanoate). On the other hand, ethyl propanoate and diacetyl presented a negative correlation with negative attributes, such as undesirable $(\mathrm{r}=-0.97)$, herbaceous and vegetal $(\mathrm{r}=-0.95)$ aromas, although there was no significant difference among undesirable and vegetal aroma for $\mathrm{C} 1-\mathrm{C} 5$ wines. On the other hand, the lower concentrations of ethyl propanoate and diacetyl occurred in C3/C5 wines, where the herbaceous aroma was higher than in C1/C4 wines (Table 2). Acetoin (3-hydroxy-2-butanone, region $\mathrm{K}$, green $\mathrm{r}=0.92$ ) also showed high and positive correlation with aroma of dry fruits. Its concentration in $\mathrm{C} 1$ was higher than in C3-C5 wines (Table 2).

Synthesis of both diacetyl and acetoin depends on the concentration of sugars in the grapes, which is related to the sun exposure and grape maturation degree. ${ }^{28}$ Researchers reported an increase in the sugar concentration of grapes when the vineyards were less vigorous ${ }^{29}$ and when defoliation treatments were performed (greater exposure of the bunches to sunlight). ${ }^{28} \mathrm{C} 1$ wine was elaborated from grapes cultivated in a vineyard with lower vegetative vigor (1.2 ratio of branches/bud) and with a less clayey soil (acrisol type) than the other vineyards. It also presented a row orientation (Northwest-Southeast (NW-SE)) favorable to solar radiation in grape clusters (Table 1). These positive characteristics of this vineyard can justify the better sensorial and volatile profile of $\mathrm{C} 1$ wine in comparison with the others.

Some compounds showed high and negative relationship with positive aroma attributes. Benzaldehyde (Tables 2 and S4) presented such a relation with aromatic intensity, aroma of red and dry fruits and alcoholic aroma $(r=-0.80,-0.80,-0.82,-0.87$, respectively). Consequently, the trend in this case means that the lower the concentration of benzaldehyde in wine, the higher these positive attributes will be. It has been already mentioned that benzaldehyde (almond) belongs to odoriferous region $\mathrm{N}$ and coelutes with ethyl-3-hydroxy-butanoate (fruity, floral), although the odor of both compounds in this chromatographic band were designated differently from the aroma of the individual compounds: green, leaf aroma. Some others have described the odor of benzaldehyde in different matrices as bitter almonds, burnt sugar, cherry, malt, and roasted pepper (Table S4, SI section). In fact, explaining the role of benzaldehyde in this particular situation is challenging and deserves further investigation. In addition, 1,2-dihydro-1,1,6-trimethyl-naphthalene (region U, TDN, rancid odor), known to develop a negative kerosene-like odor in aged wines, ${ }^{30}$ showed a negative and high relationship with alcoholic aroma $(\mathrm{r}=-0.82)$. Furthermore, these two compounds indicated a positive and high relationship with herbaceous/vegetal aroma $(r=0.87 / 0.82$ and $0.98 / 0.92$ for benzaldeyde and TDN, respectively, Table 3). These attributes may have 
Table 3. Spearman correlation between aroma attributes and odoriferous compounds presented in Cabernet Sauvignon wines produced in 2014 with grapes harvested from five vineyards in Campanha Gaúcha (C1-C5), Brazil. Experimental conditions are described in "Characterization of the wines sensory profile using sensory descriptive analysis" and "Determination of volatile profile" sub-sections. Numbers designated to each one of the compounds are the same as in Table S4 and "x" designates the number of the coelution (Co" $\mathrm{x}$ "). Capital letters indicate the odoriferous regions presented in Table 2

\begin{tabular}{|c|c|c|c|c|c|c|c|c|c|}
\hline No. & Spearman correlation & $\begin{array}{l}\text { Aromatic } \\
\text { intensity }\end{array}$ & $\begin{array}{l}\text { Aroma of } \\
\text { red fruits }\end{array}$ & $\begin{array}{l}\text { Aroma of } \\
\text { dry fruits }\end{array}$ & $\begin{array}{l}\text { Alcoholic } \\
\text { aroma }\end{array}$ & $\begin{array}{l}\text { Spices } \\
\text { aroma }\end{array}$ & $\begin{array}{c}\text { Herbaceous } \\
\text { aroma }\end{array}$ & $\begin{array}{c}\text { Undesirable } \\
\text { aroma }\end{array}$ & $\begin{array}{l}\text { Vegetal } \\
\text { aroma }\end{array}$ \\
\hline 81 & ethyl propanoate (A) & 0.98 & 0.98 & 0.95 & 0.95 & 0.55 & -0.95 & -0.97 & -0.95 \\
\hline 141 & diacetyl [2,3-butanedione] (B) & 0.98 & 0.98 & 0.95 & 0.95 & 0.55 & -0.95 & -0.97 & -0.95 \\
\hline 86 & ethyl butanoate (C) & -0.10 & -0.10 & 0.36 & -0.15 & -0.10 & 0.15 & -0.21 & -0.15 \\
\hline 18 & 1-propanol (D, Co1) & -0.40 & -0.40 & 0.05 & -0.36 & -0.67 & 0.36 & 0.16 & 0.05 \\
\hline 88 & ethyl isovalerate $(\mathrm{E})$ & 0.15 & 0.15 & 0.37 & 0.29 & -0.40 & -0.29 & -0.27 & -0.50 \\
\hline 20 & isobutyl alcohol (F) & 0.10 & 0.10 & -0.36 & 0.15 & 0.10 & -0.15 & 0.21 & 0.15 \\
\hline 90 & isoamyl acetate $(\mathrm{G})$ & 0.30 & 0.30 & 0.67 & 0.21 & 0.31 & -0.21 & -0.58 & -0.41 \\
\hline 24 & 1-butanol (H) & -0.10 & -0.10 & -0.53 & 0.03 & 0.00 & -0.03 & 0.35 & 0.34 \\
\hline 93 & ethyl hexanoate $(\mathrm{J})$ & -0.10 & -0.10 & 0.36 & -0.15 & -0.10 & 0.15 & -0.21 & -0.15 \\
\hline 145 & acetoin $(\mathrm{K}, \mathrm{Co} 8)$ & 0.67 & 0.67 & 0.92 & 0.55 & 0.53 & -0.55 & -0.87 & -0.71 \\
\hline 103 & ethyl octanoate (L, Co11) & -0.50 & -0.50 & -0.05 & -0.62 & -0.21 & 0.62 & 0.21 & 0.31 \\
\hline 174 & $p$-cymenene $(\mathrm{L}, \mathrm{Co} 11)$ & 0.41 & 0.41 & 0.76 & 0.26 & 0.34 & -0.26 & -0.65 & -0.53 \\
\hline 104 & isopentyl hexanoate (M, Co12) & -0.67 & -0.67 & -0.23 & -0.69 & -0.57 & 0.69 & 0.42 & 0.34 \\
\hline 74 & benzaldehyde (N, Co15) & -0.80 & -0.80 & -0.82 & -0.87 & -0.10 & 0.87 & 0.79 & 0.98 \\
\hline 106 & ethyl 2-hydroxy-butanoate ( $\mathrm{N}, \mathrm{Co} 15)$ & -0.29 & -0.29 & 0.00 & -0.15 & -0.74 & 0.15 & 0.15 & -0.15 \\
\hline 50 & 2,3-butanediol $(\mathrm{O})$ & 0.10 & 0.10 & 0.41 & -0.10 & 0.36 & 0.10 & -0.32 & -0.10 \\
\hline 154 & $\gamma$-butyrolactone $(\mathrm{Q}, \mathrm{Co} 18)$ & -0.30 & -0.30 & -0.67 & -0.15 & -0.21 & 0.15 & 0.53 & 0.46 \\
\hline 138 & ethyl 2-furoate (Q, Co18) & 0.40 & 0.40 & 0.15 & 0.56 & 0.05 & -0.56 & -0.26 & -0.36 \\
\hline 112 & ethyl decanoate (R, Co19) & -0.50 & -0.50 & -0.05 & -0.62 & -0.21 & 0.62 & 0.21 & 0.31 \\
\hline 4 & butanoic acid (R, Co19) & 0.60 & 0.60 & 0.67 & 0.67 & -0.15 & -0.67 & -0.58 & -0.87 \\
\hline 178 & menthol (R, Co19) & 0.35 & 0.35 & 0.73 & 0.36 & -0.18 & -0.36 & -0.56 & -0.73 \\
\hline 115 & diethyl butanedioate (S, Co21) & 0.00 & 0.00 & 0.15 & -0.21 & 0.36 & 0.21 & -0.11 & 0.10 \\
\hline 5 & isovaleric acid ( $\mathrm{S}, \mathrm{Co} 21)$ & 0.10 & 0.10 & -0.36 & 0.15 & 0.10 & -0.15 & 0.21 & 0.15 \\
\hline 196 & 3-(methylthio)-1-propanol (T, Co22) & 0.70 & 0.70 & 0.72 & 0.67 & 0.67 & -0.67 & -0.79 & -0.56 \\
\hline 57 & 2-undecanol (T, Co22) & 0.00 & 0.00 & 0.15 & -0.21 & 0.36 & 0.21 & -0.11 & 0.10 \\
\hline 180 & isopiperitone ( $\mathrm{T}, \mathrm{Co} 22)$ & 0.35 & 0.35 & 0.18 & 0.54 & -0.36 & -0.54 & -0.19 & -0.54 \\
\hline 183 & $\begin{array}{l}\text { 1,2-dihydro-1,1,6-trimethyl- } \\
\text { naphthalene (TDN) (U) }\end{array}$ & -0.72 & -0.72 & -0.76 & -0.82 & -0.03 & 0.82 & 0.73 & 0.92 \\
\hline 119 & diethyl pentanedioate $(\mathrm{V})$ & 0.35 & 0.35 & 0.18 & 0.54 & -0.36 & -0.54 & -0.19 & -0.54 \\
\hline 125 & 2-phenylethyl acetate (W, Co26) & 0.50 & 0.50 & 0.21 & 0.41 & 0.56 & -0.41 & -0.32 & -0.21 \\
\hline 187 & $\beta$-damascenone (W, Co26) & 0.10 & 0.10 & 0.41 & -0.10 & 0.36 & 0.10 & -0.32 & -0.10 \\
\hline 156 & $\gamma$-octalactone $(\mathrm{X})$ & 0.20 & 0.20 & 0.36 & 0.36 & -0.46 & -0.36 & -0.26 & -0.56 \\
\hline 64 & 1-dodecanol (Y) & -0.67 & -0.67 & -0.37 & -0.53 & -0.92 & 0.53 & 0.54 & 0.24 \\
\hline
\end{tabular}

Very weak (almost none) correlation: $0.10 \leq \mathrm{r}$; moderate correlation: $0.50 \leq \mathrm{r}<0.80$; strong correlation: $0.80 \leq \mathrm{r}<1.00$; perfect correlation: $\mathrm{r}=1.00 .^{23}$ The values in bold showed $r>0.80$ (with the exception of correlations with attributes of undesirable and vegetal that do not present significant difference).

negatively influenced the aroma of $\mathrm{C} 3$ and $\mathrm{C} 5$ wines that showed a trend towards higher concentrations of benzaldehyde in comparison to $\mathrm{C} 1$ wine $(41.2,43.7$, $47.1 \mu \mathrm{g} \mathrm{L}{ }^{-1}$ in $\mathrm{C} 1, \mathrm{C} 3$ and $\mathrm{C} 5$ wines, respectively, Table 2), as well as of TDN $\left(14.3,15.0,16.5 \mu \mathrm{g} \mathrm{L}^{-1}\right.$ in $\mathrm{C} 1, \mathrm{C} 3$ and C5 wines, respectively, Table 2).
C3 and C5 vineyards present adverse characteristics for the development of grapes, such as lower solar radiation in grape clusters and high vegetative vigor when compared to $\mathrm{C} 1$ vineyard. Solar orientation of $\mathrm{C} 3$ vineyard rows is East-West (E-W) and C5 presents high vegetative vigor (1.8 ratio of branches/bud). The low stage of maturity at the 
time of grape harvest of $\mathrm{C} 3$ and $\mathrm{C} 5$ vineyards $(17.4,17.8$, and $19.0^{\circ}$ Brix for $\mathrm{C} 3, \mathrm{C} 5$, and $\mathrm{C} 1$ vineyards, respectively, Table S3, SI section) possibly confirms these negative results, as lower ripening degree is associated with lower sugar levels, which negatively influence the formation of compounds that impart pleasant scents, during wine fermentation. Heymann et al..$^{31}$ observed that Cabernet Sauvignon wines made from grapes with lower sugar content (lower concentration of soluble solids) showed higher sourness and had more vegetative flavors than wines from grapes with higher concentration of sugars. These negative characteristics of the $\mathrm{C} 3 / \mathrm{C} 5$ vineyards may justify the less attractive sensory character of their resulting wines and the presence of volatile compounds that impair their aroma when compared to $\mathrm{C} 1$ wine. The other volatile compounds presented moderate $(0.50 \leq \mathrm{r}<0.80)$ to very weak $(0.10 \leq \mathrm{r})$ correlation with aroma attributes.

\section{Correlation between appearance and taste and mouth sensations attributes with the phenolic compounds}

The concentrations of the phenolic classes are shown in Table 4. Anthocyanins presented the highest mean concentration (102.0 $\mathrm{mg} \mathrm{L}^{-1}$, Table 4) and were followed by phenolic acids $\left(67.1 \mathrm{mg} \mathrm{L}^{-1}\right)$. The lowest average concentrations were found for flavanols, flavonols and stilbenes (45.1, 47.0 and $0.4 \mathrm{mg} \mathrm{L}^{-1}$, respectively) in Cabernet Sauvignon wines from Campanha Gaúcha region. The concentrations of phenolics of wines may vary according to the conditions of the vineyard, such as soil types, environmental climate conditions, vineyard management techniques, etc. ${ }^{5,31-35}$

Table 5 shows the Spearman correlation ( $r$ ) between appearance and taste attributes and phenolic compounds. The high correlations $(r>0.80)$ (Table 5) and the samples with statistically different concentrations (Table 4) that may explain these correlations are commented as follows. Phenolic acids presented high correlation (0.82) with smell and taste harmony and $\mathrm{C} 1$ presented high levels of these compounds $\left(71.4 \mathrm{mg} \mathrm{L}^{-1}\right)$ than $\mathrm{C} 3\left(59.3 \mathrm{mg} \mathrm{L}^{-1}\right)$ and $\mathrm{C} 5\left(64.3 \mathrm{mg} \mathrm{L}^{-1}\right)$. Flavonols and stilbenes showed high correlation values $(r>0.80)$ with color (intensity and red-purple tonality), gustatory persistence, smell and taste harmony. The concentration of flavonols in C1 $\left(51.8 \mathrm{mg} \mathrm{L}^{-1}\right)$ was statistically higher than in $\mathrm{C} 3$ and C5 (45.9, $34.6 \mathrm{mg} \mathrm{L}^{-1}$, respectively). Stilbenes also presented higher concentration in $\mathrm{C} 1\left(0.6 \mathrm{mg} \mathrm{L}^{-1}\right)$ than in $\mathrm{C} 3\left(0.3 \mathrm{mg} \mathrm{L}^{-1}\right)$. Interestingly, anthocyanins

Table 4. Phenolic compounds content in Cabernet wines obtained from different vineyards of the Campanha Gaúcha region, according to chemical classes. Experimental conditions are described in "Determination of phenolic compounds by high performance liquid chromatography-diode array detectorfluorescence detector (LC-DAD-FLD)" sub-section

\begin{tabular}{lcccccc}
\hline Class / $\left(\mathrm{mg} \mathrm{L}^{-1}\right)$ & $\mathrm{C} 1$ & $\mathrm{C} 2$ & $\mathrm{C} 3$ & $\mathrm{C} 4$ & $\mathrm{C} 5$ & Mean \\
\hline Flavanols & $39.1 \pm 0.2 \mathrm{~b}$ & $50.2 \pm 0.4 \mathrm{a}$ & $42.8 \pm 0.3 \mathrm{~b}$ & $51.3 \pm 0.2 \mathrm{a}$ & $42.0 \pm 0.1 \mathrm{~b}$ & $45.1 \pm 5.4$ \\
Flavonols & $51.8 \pm 0.3 \mathrm{a}$ & $51.5 \pm 0.2 \mathrm{a}$ & $45.9 \pm 0.3 \mathrm{~b}$ & $51.4 \pm 0.3 \mathrm{a}$ & $34.6 \pm 0.1 \mathrm{c}$ & $47.0 \pm 7.4$ \\
Anthocyanins & $105.7 \pm 1.5 \mathrm{ab}$ & $113.6 \pm 2.2 \mathrm{a}$ & $90.5 \pm 1.1 \mathrm{c}$ & $106.1 \pm 0.3 \mathrm{ab}$ & $94.1 \pm 0.3 \mathrm{bc}$ & $102.0 \pm 9.5$ \\
Phenolic acids & $71.4 \pm 0.5 \mathrm{a}$ & $72.7 \pm 0.4 \mathrm{a}$ & $59.3 \pm 0.6 \mathrm{c}$ & $67.8 \pm 0.2 \mathrm{ab}$ & $64.3 \pm 0.2 \mathrm{bc}$ & $67.1 \pm 5.5$ \\
Stilbenes & $0.6 \pm 0.1 \mathrm{a}$ & $0.4 \pm 0.1 \mathrm{ab}$ & $0.3 \pm 0.1 \mathrm{~b}$ & $0.4 \pm 01 \mathrm{ab}$ & $0.4 \pm 0.1 \mathrm{ab}$ & $0.4 \pm 0.1$ \\
\hline
\end{tabular}

In the same line means showing common letter are not significantly different $(p=5 \%)$ according ANOVA and Tukey's test.

Table 5. Spearman correlation (r) between appearance and taste attributes and phenolic compounds observed in Cabernet Sauvignon wines, according to chemical classes. Experimental conditions are described in "Characterization of the wines sensory profile using sensory descriptive analysis" and "Determination of phenolic compounds by high performance liquid chromatography-diode array detector-fluorescence detector (LC-DAD-FLD)" sub-sections

\begin{tabular}{|c|c|c|c|c|c|c|c|c|c|}
\hline \multirow{2}{*}{$\begin{array}{l}\text { Spearman } \\
\text { correlation }\end{array}$} & \multicolumn{2}{|c|}{ Appearance } & \multicolumn{7}{|c|}{ Taste and mouth sensations } \\
\hline & $\begin{array}{c}\text { Color } \\
\text { intensity }\end{array}$ & $\begin{array}{c}\text { Red-purple } \\
\text { tonality }\end{array}$ & $\begin{array}{c}\text { Gustatory } \\
\text { persistence }\end{array}$ & Body & $\begin{array}{c}\text { Smell and } \\
\text { taste harmony }\end{array}$ & Astringency & Bitterness & Sourness & Sweetness \\
\hline Flavanols & -0.10 & -0.10 & -0.10 & -0.10 & -0.15 & 0.30 & 0.10 & 0.70 & -0.05 \\
\hline Flavonols & 0.80 & 0.80 & 0.80 & 0.80 & 0.87 & 0.60 & 0.30 & 0.60 & 0.20 \\
\hline Anthocyanins & 0.60 & 0.60 & 0.60 & 0.60 & 0.67 & 0.70 & 0.40 & 0.80 & -0.20 \\
\hline Phenolic acids & 0.70 & 0.70 & 0.70 & 0.70 & 0.82 & 0.60 & 0.30 & 0.60 & -0.05 \\
\hline Stilbenes & 0.89 & 0.89 & 0.89 & 0.89 & 0.92 & 0.67 & 0.67 & 0.22 & -0.46 \\
\hline
\end{tabular}

Very weak (almost none) correlation: $0.10 \leq \mathrm{r}$; moderate correlation: $0.50 \leq \mathrm{r}<0.80$; strong correlation: $0.80 \leq \mathrm{r}<1.00$; perfect correlation: $\mathrm{r}=1.00{ }^{23}$ The values in bold showed $r>0.80$ (with the exception of correlations with attributes of sweetness that do not present significant difference). 
have shown a high correlation with sourness $(r=0.80$, Table 5) and $\mathrm{C} 1$ wine presented higher concentration of this phenolic class $\left(105.7 \mathrm{mg} \mathrm{L}^{-1}\right)$ than $\mathrm{C} 3\left(90.5 \mathrm{mg} \mathrm{L}^{-1}\right)$ and $\mathrm{C} 5\left(94.1 \mathrm{mg} \mathrm{L}^{-1}\right)$ wines. A high correlation between sourness and anthocyanins is in accordance with literature..$^{34}$ In addition, anthocyanins may also be related to astringency, even though the correlation between them was less intense $(r=0.70) .{ }^{35}$ According to Noble, ${ }^{36}$ red wine astringency and bitterness are provided primarily by flavanols and tannins. However, flavanols under study have not shown significant correlation with astringency, neither with bitterness.

Both flavonols and stilbenes have been reported to influence wine color and red purple tonality because these compounds may act as anthocyanin copigments. ${ }^{34,37}$ Copigmentation effect is the formation of complexes between anthocyanins and other flavonoids, phenolic acids, stilbenes, proteins, amino acids or polysaccharides. ${ }^{35}$ Gaudette and Pickering ${ }^{37}$ showed evidence of a color shift to a more intense color for Cabernet Sauvignon with increasing trans-resveratrol concentration, although no change was observed in the wine flavor profile. Such an effect may also be explained by copigmentation due to fortification with trans-resveratrol.

The relation of smell/taste harmony with phenolic acids was for the first time reported in red wines as well as the association of gustatory persistence and smell/taste harmony with flavonols. Color intensity, body, astringency, and bitterness of Pinot Noir ${ }^{32}$ and Istrian Malvasia ${ }^{33}$ wines have been associated with anthocyanins, flavanols and flavonols, taking into consideration compound classes and also some individual phenolic compounds. The intensity of these sensory attributes has already been linked to grape solar exposure due to practices such as leaf removal and pruning. ${ }^{32,33}$ Solar irradiation may induce changes in the biosynthesis of phenolic compounds in grapes and consequently in the wines of these corresponding grapes. ${ }^{5,38,39}$ However, the link between vineyard management practices, wine phenolic compounds and sensory attributes through Spearman correlation (r) is reported for the first time. Interestingly, $\mathrm{C} 1$ wine was produced from the vineyard that received the highest solar radiation due to its lower vegetative vigor, which is a consequence of favorable solar orientation (NW-SE), in addition to an acrisol soil (less clayey) and an appropriate bunch/bud ratio: 1.2, as shown in Table 1. A less clayey soil (acrisol), with low content of organic matter $\left(11 \mathrm{~g} \mathrm{~kg}^{-1}\right)$ is in accordance with less vegetative vigor, which corroborates with results found. ${ }^{3}$ As previously mentioned, $\mathrm{C} 1$ wine showed the highest concentration of some phenolic compound classes (flavonols stilbenes, anthocyanins and phenolic acids) positively related to appearance (color intensity and red-purple tonality) and taste and mouth sensations.

In contrast, $\mathrm{C} 3$ wine showed the lowest concentration of some phenolics (anthocyanins, phenolic acids, stilbenes) compared to $\mathrm{C} 1$ (Table 4). This wine was produced from a vineyard with an E-W solar orientation, which received the least amount of solar radiation among the vineyards (NW-SE, N-S, Northeast-Southwest (NE-SW), N-S row orientation to $\mathrm{C} 1, \mathrm{C} 2, \mathrm{C} 4$ and $\mathrm{C} 5$, respectively, Table 1). In addition, grapes of $\mathrm{C} 3$ vineyard showed the lowest ripeness level $\left(19.0,19.0,17.4,18.3,17.8{ }^{\circ}\right.$ Brix, corresponding to $\mathrm{C} 1, \mathrm{C} 2, \mathrm{C} 3, \mathrm{C} 4$ and $\mathrm{C} 5$ wines, respectively, Table S3). Accordingly, leaf removal and consequently higher solar incidence resulted in higher concentrations of flavonols (they were higher in $\mathrm{C} 1$ than in $\mathrm{C} 3$ ) and also in several varietal grapes and wines, such as higher quercetin$3-\beta$-D-glucoside concentrations in Pinot Noir grapes with $50 \%$ (626 $\mathrm{mg} \mathrm{L}^{-1}$ ) and 100\% (1003 $\mathrm{mg} \mathrm{L}^{-1}$ ) of leaf removal than in grapes without leaf removal $\left(199 \mathrm{mg} \mathrm{L}^{-1}\right) .{ }^{1}$ Cortell and Kennedy ${ }^{38}$ have demonstrated that the lack of solar incidence on grapes might cause a reduction in the sum of concentrations of flavonols $\left(45 \mathrm{mg} \mathrm{L}^{-1}\right)$ of Pinot Noir grapes compared to grapes exposed to sun $\left(111 \mathrm{mg} \mathrm{L}^{-1}\right)$. Moreover, higher concentration of anthocyanins (491 $\mathrm{mg} \mathrm{L}^{-1}$ ) was also observed in Nero di Troia wines whose vineyard had its leaves removed in the area of grape clusters than in wines obtained from a vineyard that did not have its leaves removed $\left(411 \mathrm{mg} \mathrm{L}^{-1}\right){ }^{27} \mathrm{In}$ line with Cantos et al. ${ }^{40}$ solar radiation can cause stress to the plant resulting in an increase of trans-resveratrol content, as a defense strategy. Early defoliation improved the phenolic composition of Tempranillo wines also by favoring the accumulation of hydroxycinnamic acids, flavonols and anthocyanins, thus enhancing wine quality in terms of color and sensory properties..$^{28}$ Accordingly, other researchers have also reported that the content of anthocyanins in wines increased with less vigorous vineyards ${ }^{39}$ and that leaf removal in the fruit zone increased the level of anthocyanins in final wine. ${ }^{5}$

Concerning stilbenes, the Malbec grapes also presented higher concentration of trans-resveratrol when they were under sunlight with full UV-B radiation $\left(4.2 \mathrm{mg} \mathrm{kg}^{-1}\right)$ in comparison with grapes that had the incidence of filtered UV-B $\left(3.0 \mathrm{mg} \mathrm{kg}^{-1}\right)$. $^{41}$

The final assumption about correlations between phenolic compounds and sensory attributes has to be confirmed with wine model solutions with these specific compounds, however, the present results represent an important advance in this area and may facilitate finding more specific directions for further research. 


\section{Conclusions}

A comprehensive analytical approach encompassing LC-DAD-FLD, GC-MS, GC × GC-TOFMS, GC-O-Osme, and DA was essential to point that Cabernet Sauvignon wine produced from grapes of vineyard $\mathrm{C} 1$ of Campanha Gaúcha region showed the best quality among wines of five vineyards spread over this same region, presenting the highest concentration of volatiles (mainly ethyl propanoate, diacetyl and acetoin) that were statistically correlated with positive sensory attributes and to vineyard field parameters. GC $\times$ GC-TOFMS was fundamental to prevent misidentification of compounds that were responsible for these correlations. $\mathrm{C} 1$ wine also showed the highest concentration of some phenolic compound classes (flavonols, stilbenes, anthocyanins and phenolic acids) that were positively related to appearance (color intensity and red-purple tonality), taste and mouth sensations. A convenient solar exposure, a canopy management that resulted in lower vegetative growth, and a less clayey soil with lower organic matter might be main responsible parameters for $\mathrm{C} 1$ wine better quality. In addition, Cabernet Sauvignon wines of this emerging region of fine wines were described in terms of 197 volatiles and five classes of phenolic compounds for the first time and their characterization helps to support the achievement of geographical indication and future denomination of origin.

These results open a path for a more comprehensive and detailed investigation of wine quality with the aim of pursuing improvements in vineyard field management guided by beneficial or deleterious influence of volatile and/or phenolic compounds in wine sensory attributes. The high degree of detail of this approach will certainly facilitate the improvement of field management to achieve a better grape and wine quality. However, further studies with model solutions and mixtures of model compounds should ensure the individual contribution of each volatile compound, as well as account for synergistic effects.

\section{Supplementary Information}

Supplementary data (geographical location, climate conditions, physico-chemical parameters, volatile compounds, mean score of descriptive attributes, aroma compounds) are available free of charge at http://jbcs.sbq.org.br as PDF file.

\section{Acknowledgments}

The authors thank the National Council for Scientific and Technological Development (Conselho Nacional de
Desenvolvimento Científico e Tecnológico, CNPq), the Coordination for the Improvement of Higher Education Personnel (Coordenação de Aperfeiçoamento de Pessoal de Nível Superior, CAPES), and the Research Support Foundation of Rio Grande do Sul (Fundação de Amparo à Pesquisa do Estado do Rio Grande do Sul, FAPERGS) for financial support and scholarships for Karine P. Nicolli (CAPES AUX-PE-PROEX 587/2017) and Claudia A. Zini (CNPq Pq 1D 306067/2016-1). We also acknowledge SIBRATEC/FINEP/FAPEG (01.13.0210.00, Project IPCampanha) for supporting sampling, transportation, and vinification. Scientific discussions with Prof João Henrique Zimnoch dos Santos were appreciated.

\section{Author Contributions}

Karine P. Nicolli prepared the samples and has run the chromatographic analyses, except for the LC-DAD. She also participated in the sensory panel of the DA and also of the GC-O-Osme. She was in charge of data processing and of the writing of this manuscript; Aline C. T. Biasoto was in charge of the training of the sensory panels of DA and also of the GC-O-Osme. Aline also helped in discussing results and reviewing the manuscript; Celito C. Guerra was in charge of planning and performing the microvinification experiments in the lab and also responsible for the physicochemical analyses; Henrique P. dos Santos was responsible for planning and performing the vineyard experiments. Sampling of grapes was also done by him; Juliane E. Welke was the research collaborator in all steps of this work, since the beginning of this project until de end; Luiz C. Correa collaborated in the planning of the analyses of phenols and has run the LC-DAD analyses; Claudia A. Zini was the supervisor of all the work since the planning of the experiments from the original idea until the final part of writing the manuscript. She has participated in the majority of the experimental steps, supervising results and discussions.

\section{References}

1. Feng, H.; Yuan, F.; Skinkis, P. A.; Qian, M. C.; Food Chem. 2015, 173, 414.

2. Hunter, J. J.; Volschenk, C. G.; Zorer, R.; Agric. For. Meteorol. 2016, 228, 104.

3. Lanyon, D. M.; Cass, A.; Hansen, D.; CSIRO Land and Water Technical Report No. 34/04; CSIRO: Glen Osmond, Australia, 2004.

4. Keller, M.; The Science of Grapevines: Anatomy and Physiology; Academic Press: London, England, 2010.

5. Feng, H.; Skinkis, P. A.; Qian, M. C.; Food Chem. 2017, 214, 736. 
6. Moreno, D.; Valdés, E.; Uriarte, D.; Gamero, E.; Talaverano, I.; Vilanova, M.; Food Res. Int. 2017, 98, 50.

7. Robinson, A. L.; Boss, P. K.; Heymann, H.; Solomon, P. S.; Trengove, R. D.; J. Agric. Food Chem. 2011, 59, 3273.

8. Vilanova, M.; Diago, M. P.; Genisheva, Z.; Oliveira, J. M.; Tardaguila, J.; J. Sci. Food Agric. 2012, 92, 935.

9. Nicolli, K. P.; Biasoto, A. C. T.; Souza-Silva, É. A.; Guerra, C. C.; dos Santos, H. P.; Welke, J. E.; Zini, C. A.; Food Chem. 2018, 243, 103.

10. https://www.embrapa.br/uva-e-vinho/cadastro-viticola, accessed in December 2019.

11. https://www.embrapa.br/en/busca-de-noticias/-/ noticia/30674920/indicacao-geografica-de-vinhos-finos-dacampanha-gaucha-a-caminho, accessed in December 2019.

12. Chin, S.-T. T.; Eyres, G. T.; Marriott, P. J.; Food Chem. 2015, $185,355$.

13. Villière, A.; Arvisenet, G.; Lethuaut, L.; Prost, C.; Sérot, T.; Food Chem. 2012, 131, 1561.

14. Stone, H.; Sidel, J.; Oliver, S.; Woolsey, A.; Singleton, R. C. In Descriptive Sensory Analysis in Practice; Gacula Jr., M. C., ed.; Food \& Nutrition Press: Trumbull, USA, 1974, 23.

15. Natividade, M. M. P.; Corrêa, L. C.; de Souza, S. V. C.; Pereira, G. E.; Lima, L. C. O.; Microchem. J. 2013, 110, 665.

16. International Union of Soil Sciences (IUSS) Working Group World Reference Base (WRB); World Soil Resources Reports No. 106; Food and Agriculture Organization of the United Nations (FAO): Rome, 2015.

17. Blouin, J.; Peynaud, E.; Connaissance et Travail du Vin, $5^{\text {th }}$ ed.; Duned: Paris, France, 2012.

18. Ministério da Agricultura, Pecuária e Abastecimento (MAPA); Portaria No. 229, de 25 de Outubro de 1988, DOU 31/10/1988, available at http://www.agricultura.gov.br/assuntos/vigilanciaagropecuaria/ivegetal/bebidas-arquivos/portaria-no-229-de-25de-outubro-de-1988.pdf/view, accessed in December 2019.

19. Moskowitz, H. R.; Product Testing and Sensory Evaluation of Foods: Marketing and R\&D Approaches; Food \& Nutrition Press: Westport, USA, 1983.

20. Ministério da Saúde; Conselho Nacional da Saúde; Resolução No. 466; Aprova Diretrizes e Normas Regulamentadoras de Pesquisas Envolvendo Seres Humanos; Diário Oficial da União (DOU), Brasília, de 12/12/12.

21. Welke, J. E.; Zanus, M.; Lazarotto, M.; Schmitt, K. G.; Zini, C. A.; J. Braz. Chem. Soc. 2012, 23, 678.

22. SPSS Statistics, version 15.0; IBM, Chicago, USA, 2003.
23. Granato, D.; Calado, V. M. A.; Jarvis, B.; Food Res. Int. 2014, $55,137$.

24. Weldegergis, B. T.; Crouch, A. M.; Górecki, T.; de Villiers, A.; Anal. Chim. Acta 2011, 701, 98.

25. http://www.thegoodscentscompany.com/data/rw1014671.html, accessed in December 2019.

26. Clarke, R. J.; Bakker, J.; Wine Flavour Chemistry; Clarke, R. J.; Bakker, J., eds.; Blackwell Publishing Ltd.: Oxford, UK, 2004.

27. Biasoto, A. C. T.; Netto, F. M.; Marques, E. J. N.; da Silva, M. A. A. P.; Food Res. Int. 2014, 62, 456.

28. Baiano, A.; de Gianni, A.; Previtali, M. A.; Del Nobile, M. A.; Novello, V.; de Palma, L.; Food Res. Int. 2015, 75, 260.

29. Cortell, J. M.; Halbleib, M.; Gallagher, A. V.; Righetti, T. L.; Kennedy, J. A.; J. Agric. Food Chem. 2005, 53, 5798.

30. Mayr, C. M.; Geue, J. P.; Holt, H. E.; Pearson, W. P.; Jeffery, D. W.; Francis, I. L.; J. Agric. Food Chem. 2014, 62, 4528.

31. Heymann, H.; Licalzi, M.; Conversano, M. R.; Bauer, A.; Skogerson, K.; Matthews, M.; S. Afr. J. Enol. Vitic. 2013, 34, 86.

32. Cortell, J. M.; Sivertsen, H. K.; Kennedy, J. A.; Heymann, H.; Am. J. Enol. Vitic. 2008, 59, 1.

33. Rescic, J.; Mikulic-Petkovsek, M.; Rusjan, D.; J. Sci. Food Agric. 2016, 96, 4724.

34. Moreno-Arribas, M. V.; Polo, M. C.; Wine Chemistry and Biochemistry, $1^{\text {st }}$ ed.; Moreno-Arribas, M. V.; Polo, M. C., eds.; Springer: New York, USA, 2009, 249.

35. Ribéreau-Gayon, P.; Glories, Y.; Maujean, A.; Dubourdieu, D.; Handbook of Enology, vol. 2, $2^{\text {nd }}$ ed.; John Wiley \& Sons Ltd.: Chichester, England, 2006.

36. Noble, A. C.; Physiol. Behav. 1994, 56, 1251.

37. Gaudette, N. J.; Pickering, G. J.; Aust. J. Grape Wine Res. 2011, $17,249$.

38. Cortell, J. M.; Kennedy, J. A.; J. Agric. Food Chem. 2006, 54, 8510.

39. Song, J.; Smart, R. E.; Dambergs, R. G.; Sparrow, A. M.; Wells, R. B.; Wang, H.; Qian, M. C.; Food Chem. 2014, 153, 52.

40. Cantos, E.; Garcia-Viguera, C.; de Pascual-Teresa, S.; TomasBarberan, F. A.; J. Agric. Food Chem. 2000, 48, 4606.

41. Berli, F.; D’Angelo, J.; Cavagnaro, B.; Bottini, R.; Wuilloud, R.; Silva, M. F.; J. Agric. Food Chem. 2008, 56, 2892.

Submitted: September 18, 2019 Published online: December 19, 2019 